\title{
Vaccine-Derived
}

\section{Polioviruses and the Endgame Strategy for Global Polio Eradication*}

\author{
Olen M. Kew, ${ }^{1}$ Roland W. Sutter, ${ }^{2}$ \\ Esther M. de Gourville, ${ }^{2}$ Walter R. Dowdle, ${ }^{3}$ \\ and Mark A. Pallansch ${ }^{1}$
}

\footnotetext{
${ }^{1}$ Division of Viral and Rickettsial Diseases, National Center for Infectious Diseases, Centers for Disease Control and Prevention, Atlanta, Georgia 30333; email: omk1@cdc.gov; map1@cdc.gov

${ }^{2}$ World Health Organization, CH-1211, Geneva 27, Switzerland; email: sutterr@who.int; degourvillee@who.int

${ }^{3}$ Task Force for Child Survival and Development, Decatur, Georgia 30030; email: wdowdle@taskforce.org
}

Annu. Rev. Microbiol.

2005. 59:587-635

The Annual Review of Microbiology is online at micro.annualreviews.org

doi: 10.1146/

annurev.micro.58.030603.123625

Copyright (c) 2005 by Annual Reviews. All rights reserved

*The U.S. Government has the right to retain a nonexclisive, royalty-free license in and to any copyright covering this paper.

$0066-4227 / 05 / 1013-$ $0587 \$ 20.00$

\section{Key Words}

poliomyelitis, attenuation of neurovirulence, oral poliovirus vaccine
Abstract
As the global eradication of wild poliovirus nears, the World Health Organization (WHO) is addressing challenges unprecedented in public health. The live, attenuated oral poliovirus vaccine (OPV), used for more than four decades to interrupt poliovirus transmission, and the vaccine of choice for developing countries, is genetically un- stable. Reversion of the small number of substitutions conferring the attenuated phenotype frequently occurs during OPV replica- tion in humans and is the underlying cause of the rare cases of vaccine-associated paralytic poliomyelitis (VAPP) in OPV recipi- ents and their close contacts. Whereas VAPP has long been recog- nized, two other adverse events have been identified more recently: (a) long-term excretion of highly evolved vaccine-derived po- lioviruses (VDPVs) in persons with primary immunodeficiencies, and $(b)$ polio outbreaks associated with circulating VDPVs in ar- eas with low rates of OPV coverage. Developing a posteradication strategy to minimize the risks of VDPV emergence and spread has become an urgent $\mathrm{WHO}$ priority. 


\section{Contents}

INTRODUCTION.............. 588

POLIOVIRUSES AND

POLIOMYELITIS ............ 589

Polioviruses.................... 589

Poliomyelitis .................. 590

POLIOVIRUS VACCINES ........ 591

Inactivated Poliovirus Vaccine..... 591

Oral Poliovirus Vaccine.......... 592

Genetic Determinants of

Attenuation of the Sabin OPV

Strains ................... 593

The Remarkable Safety Record of OPV ................. 596

Molecular Approaches Toward the Development of More Stable OPV Strains........... 596

CONTROL OF POLIO BY

IMMUNIZATION ........... 597

Developed Countries ............ 597

Developing Countries ........... 597

The Global Polio Eradication

Initiative ............... 597

VACCINE-ASSOCIATED

PARALYTIC POLIOMYELITIS . 603

VACCINE-DERIVED

POLIOVIRUSES .............. 604

Categories of VDPVs............ 604

iVDPVs from Long-Term

Poliovirus Excretors .......... 606

cVDPVs from Polio Outbreaks ... 607
Evidence of Past VDPV

Circulation................. 609

Other Possible Examples of VDPV

Circulation.................. 609

Risk Factors for cVDPV

Emergence and Spread ........ 612

Properties Shared by iVDPV and cVDPV Isolates .............. 613

Properties Distinguishing iVDPV and cVDPV Isolates .......... 613

aVDPVs Reconsidered ........... 615

Independence of iVDPVs and cVDPVs ................ 615

Laboratory Surveillance for VDPVs

The Surprising Serotype

Distribution for VDPVs

VDPVs AND THE ENDGAME

STRATEGY FOR POLIO

ERADICATION

Pre- and Posteradication

Challenges............... 617

Eradication Principles Revisited... 617

Limited Applicability of the Smallpox Model............ 618

Early Alternative Endgame Scenarios .................. 618

Current Endgame Perspectives and Challenges............ 620

CONCLUSIONS ................ 622
IPV: inactivated poliovirus vaccine

OPV: oral poliovirus vaccine

EPI: Expanded Program on Immunization

WHO: World Health Organization

\section{INTRODUCTION}

The year 2005 marks the fiftieth anniversary of the introduction of the IPV of Salk and Youngner $(149,173)$, and the promise of a world free of poliomyelitis (polio). Within 15 years that promise had been realized in most developed countries, through widespread immunization with IPV and then with the live, attenuated OPV of Albert Sabin (170), introduced in the early 1960s. From the perspective of developed industrialized countries, the conquest of polio marked one of the great- est public health triumphs of the twentieth century.

The promise had yet to be fulfilled in the developing world, where polio continued to threaten children with lifelong paralysis. The eradication of smallpox in 1977 (52) provided a major impetus to global control of vaccine-preventable diseases and set the stage for the EPI (created by WHO), which targets six vaccine-preventable infectious diseases, including polio, for worldwide control (67). The cornerstone of the EPI strategy was high 
vaccine coverage through routine immunization, and OPV was ideally suited for use in the EPI. Experience in the Americas (165), however, revealed the limitations of the routine immunization strategy for polio in tropical developing countries, and a more aggressive strategy was launched, first in the Americas in 1985 (145) and then worldwide in 1988 (207), based upon supplementing routine immunization with mass OPV campaigns.

By 2005, wild polioviruses have been brought to the brink of extinction, with a $>99 \%$ reduction in the worldwide incidence of polio since 1988 (219), the eradication of type 2 wild poliovirus (208), and the localization of endemic wild poliovirus transmission to a few countries in Africa and Asia (219). However, just as the prospects for global polio eradication brightened, long held concerns (11) arose anew about the genetic stability of the primary weapon against polio, the live, attenuated OPV (55). The spectacular success of global polio eradication had fundamentally altered the ecology of poliovirus in most of the world, as wild polioviruses were no longer in circulation and community immunity to poliovirus could be maintained only through high rates of vaccine (usually OPV) coverage. Recent reports describing highly divergent VDPVs associated with chronic poliovirus infections $(10,69,88,90,113,123$, $216,223)$ or with polio outbreaks $(84,169$, $177,226)$ underscored the earlier concerns and challenged the critical assumption that OPV-derived viruses would not long persist in the community. These findings prompted a reassessment by $\mathrm{WHO}$ of both current and future immunization strategies. Here we review the rapidly developing understanding of the biological properties of VDPVs, of the conditions permissive for their emergence and spread, and how their discovery has shaped the WHO endgame strategy for global polio eradication. VDPVs and the endgame strategy have been the subject of some recent special-theme issues of international journals $(16,221)$, of recent brief reviews $(43,69,89$,
$125,136)$, and of a continuing series of reports from WHO $(215,220,221)$.

\section{POLIOVIRUSES AND POLIOMYELITIS}

\section{Polioviruses}

Polioviruses are members of the Enterovirus genus of the family Picornaviridae (142, 156). Polioviruses are small (27 to $30 \mathrm{~nm}$ in diameter), nonenveloped viruses with capsids of icosahedral symmetry enclosing a singlestranded, positive-sense RNA genome. The genome is about $7500 \mathrm{nt}$ long and has a small protein, $\mathrm{VPg}$, covalently linked to the $5^{\prime}$ end and polyadenylated at the $3^{\prime}$ end (Figure 1). The single ORF is flanked by a long $(\sim 740 \mathrm{nt})$ 5'-UTR and a short ( $\sim 70 \mathrm{nt}) 3^{\prime}$-UTR. There are three antigenic types (serotypes 1, 2, and 3) (13), and complete genomic sequences have been determined for numerous representatives of each type, including those of the three Sabin OPV strains (196). Only the sequences encoding the capsid proteins are unique to polioviruses, as the flanking sequences are frequently exchanged by recombination with the closely related species $\mathrm{C}$ enteroviruses during circulation in nature $(15,66,84,107$, 226). The poliovirion consists of 60 copies of each of the four capsid proteins (VP1, VP2, VP3, VP4) that form a highly structured capsid shell (77). The three major proteins (VP1, VP2, VP3) share a similar basic architecture and were probably derived from a common ancestral protein. The smallest protein, $\mathrm{VP} 4$, internalized in the native virion, is formed by the cleavage of the precursor VP0 (VP4 + VP2) during the final maturation of the virion. The external surface of the poliovirion is decorated by peptide loops extending from VP1, VP2, and VP3, which form the neutralizing antigenic sites (124, 126). Three (or four) neutralizing antigenic sites have been identified by patterns of reactivity with neutralizing monoclonal antibodies, and the assignments have been confirmed by high-resolution X-ray crystallography (54,
VDPVs:

vaccine-derived polioviruses

ORF: open reading frame

UTR: untranslated region 

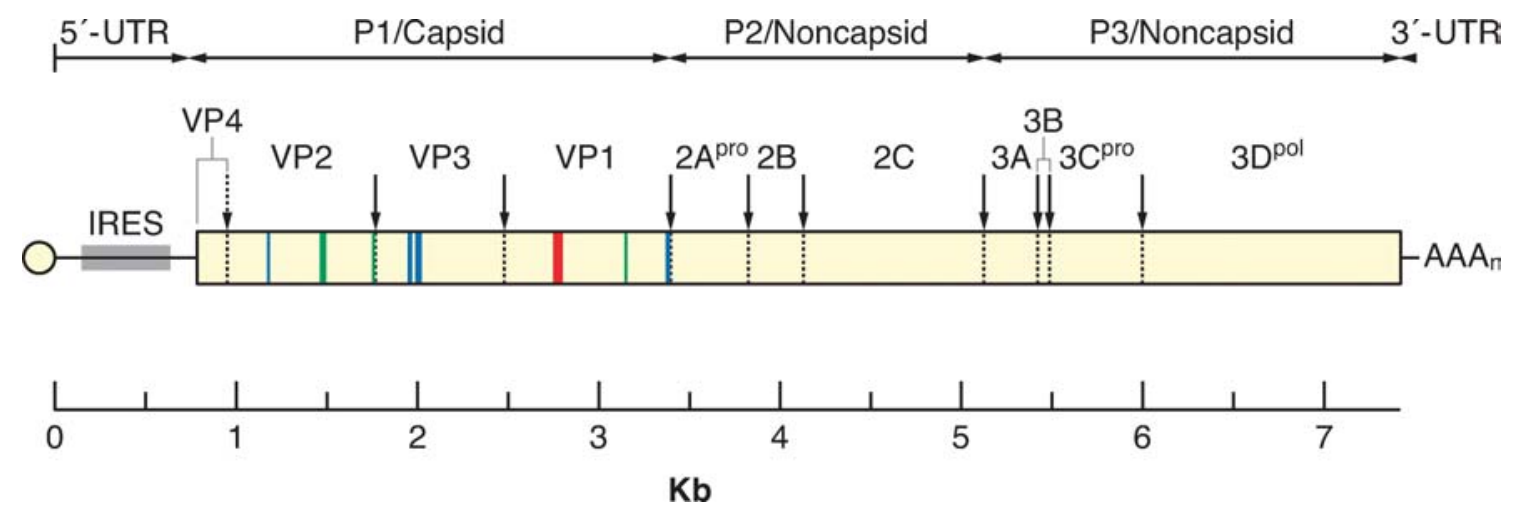

Figure 1

Schematic of the poliovirus genome. The single ORF is indicated by a rectangle, flanked by the $5^{\prime}$ - and $3^{\prime}$-UTRs; the small protein VPg (encoded by the 3B sequence interval) is covalently attached to the $5^{\prime}$-UTR and is represented by a circle at the $5^{\prime}$ end. The IRES (nucleotide positions $\sim 130$ to 600 ) in the $5^{\prime}$-UTR is shown as a shaded rectangle. A single polyprotein is translated from the ORF, which is processed by virus-encoded proteinases $2 \mathrm{~A}^{\text {pro }}$ (catalyzes cleavage between VP1 and $2 \mathrm{~A}^{\text {pro }}$; the cleavage site is indicated by a dashed arrow) and 3CD pro (catalyzes all other cleavages except the VP4/VP2 maturation cleavage; the cleavage sites are indicated by the solid arrows). Mature cleavage products are bounded by dashed lines. Protein $3 \mathrm{D}^{\mathrm{pol}}$ is an RNA-dependent RNA polymerase. Colored bars symbolize virion surface loops that form neutralizing antigenic sites 1 (red), 2 (green), and 3 (blue).

IRES: internal ribosome entry site
103, 141) (Figure 1). Neutralizing antigenic site 1 is continuous and formed by a loop in VP1; sites 2 and 3 are discontinuous and formed from loops contributed by different capsid proteins. The major type-specific differences in the capsid polypeptides primarily reside on the most surface-accessible peptide loops, which represent less than $4 \%$ of the total capsid protein (196). Although the neutralizing antigenic sites vary within each serotype, the range of variability is constrained, possibly because of steric requirements for interaction with the poliovirus receptor (72), such that all polioviruses within a serotype can be neutralized by type-specific antisera, and poliovaccines (one vaccine strain for each serotype) can induce protective immunity to all known antigenic variants.

Polioviruses attach to and enter cells via the specific poliovirus receptor on the cytoplasmic membrane, CD155, a glycoprotein of the immunoglobulin superfamily $(64,76$, 122). After entry, the genomic RNA is uncoated and translated under the control of the IRES, an element (nucleotide positions $\sim 130$ to 600 ) within the $5^{\prime}$-UTR that has a highly conserved stem-loop structure (47). The translation product is a single polypeptide, the polyprotein, which is cleaved by the virus-encoded proteinases $2 \mathrm{~A}^{\text {pro }}$ and $3 \mathrm{CD}^{\text {pro }}$ into mature viral proteins (Figure 1). Host protein synthesis is rapidly inhibited by the cleavage by $2 \mathrm{~A}^{\text {pro }}$ of the translation initiation factor eIF4G (102), required for initiation of translation of capped host messenger RNA but not for the internal initiation of translation from the poliovirus IRES (156). Infected cells show cytopathic effects within 4 to 6 hours, and release up to 10,000 infectious virus particles upon cell lysis and death.

\section{Poliomyelitis}

Pathogenesis. Only a small proportion $(<1 \%)$ of poliovirus infections of susceptible individuals result in paralytic poliomyelitis. Most infections are inapparent, although some infected people experience minor illness (fever, headache, malaise) or aseptic meningitis. Paralytic poliomyelitis is preceded by minor illness of several days and a symptom-free 
interval of 1 to 3 days, followed by acute onset of flaccid paralysis with fever. Paralysis, which is usually asymmetric, progresses within a few days and, depending upon the sites of virus replication in the central nervous system, may affect skeletal muscles (spinal poliomyelitis), respiratory muscles (bulbar poliomyelitis), or both (bulbo-spinal poliomyelitis) $(159,185)$. The loss of motor neurons is permanent and the denervated muscles atrophy.

Paralytic illness follows directly from the lytic infection of motor neurons: in the anterior horn for spinal poliomyelitis, or in the medulla for bulbar poliomyelitis $(64,159)$. Following ingestion, poliovirus replicates locally at the sites of initial virus implantation (tonsils, intestinal $M$ cells, Peyer's patches of the ileum, and the mesenteric lymph nodes). After amplification at these sites, virus enters the bloodstream and infects other tissues, occasionally including motor neurons. Local secretory IgA immunity can block poliovirus replication in tonsils and intestinal tract, and neutralizing IgG and IgM antibodies can prevent virus spread to motor neurons of the central nervous system. The host range of poliovirus is determined by the CD155 receptor, a cell-cell adhesion molecule (130a) that is found only on the cells of humans, higher primates, and Old World monkeys.

Epidemiology. Poliomyelitis is highly contagious, and wild polioviruses in endemic areas infect virtually the entire population (185). In highly endemic reservoir areas, many children are infected within the first year of life. During the endemic period in developed countries with good sanitation and in isolated populations, exposure often occurred later in life, sometimes during major outbreaks. Polio is a seasonal disease, with peak transmission in the summer and autumn and reduced transmission during winter. Seasonal differences in transmission are far less pronounced in tropical areas $(135,170)$. Paralytic attack rates vary by serotype, with the highest rates $(\sim 0.5 \%)$ associated with poliovirus type 1 and the lowest rates $(<0.05 \%)$ associated with po- liovirus type 2 (135). Poliovirus is transmitted person to person by the fecal-oral route and also by the oral-oral route, especially in areas with good sanitation and hygiene. Major risk factors for poliovirus transmission include poor sanitation and hygiene, tropical and subtropical conditions, large birth cohorts, and high population densities $(135,170)$.

\section{POLIOVIRUS VACCINES}

Two different poliovaccines have been developed, the IPV of Salk and Youngner (152, 173) and the live, attenuated OPV of Sabin (185). Both vaccines are safe and effective, each with its particular advantages and disadvantages (121), and both have played an important role in the control of poliomyelitis $(149,166)$.

\section{Inactivated Poliovirus Vaccine}

IPV was the first poliovaccine to be licensed. Its development followed several key advances in virology (152): (a) the cultivation of poliovirus in non-neural cells (49), (b) the identification of three poliovirus serotypes (13), (c) the finding that viremia precedes paralysis (78), and (d) the demonstration that administration of immune globulin protects against paralytic polio (70). IPV is prepared by formalin inactivation of three wild, virulent reference strains, Mahoney (type 1), MEF-1 (type 2), and Saukett (type 3). A less virulent type 1 strain, Brunenders (50), is used in IPV production in Sweden and Denmark. Although antigenic sites 1 (Figure 1) of types 2 and 3 are modified by formalin inactivation (53), immunization with IPV can induce high titers of neutralizing antibodies protective against all poliovirus strains. After the Cutter incident (134), conditions for IPV manufacture were modified, resulting in a reduction in the immunogenicity of IPV preparations. However, improvements in cell culture technology in the 1970s led to the development of an enhanced-potency IPV, similar in immunogenicity to the original product 
VAPP:

vaccine-associated paralytic poliomyelitis
$(152,200)$, which has replaced the secondgeneration IPV.

IPV was licensed for use in the United States, Canada, and Western Europe in 1955 and was the only poliovaccine available until licensure of OPV in 1961-1962. IPV use in the United States declined after the introduction of OPV, but it has been used continuously by some countries in Western Europe (Finland, Iceland, Sweden, and The Netherlands) and some provinces of Canada (152). In 1997, in response to the eradication of wild polioviruses in the Americas and the continuing occurrence of cases of VAPP (see below), the United States shifted from an allOPV immunization schedule to a sequential IPV/OPV schedule, which was replaced in 2000 by an all-IPV schedule $(3,152)$.

\section{Oral Poliovirus Vaccine}

Development of the Sabin OPV strains. Early attempts to produce live-virus vaccines date to the work of Jenner, who "vaccinated" with cowpox virus to protect against smallpox in the 1790s, and Pasteur, who developed an "attenuated" rabies vaccine in the 1880s (153). In the 1930s, Theiler \& Smith (195) demonstrated that an effective attenuated yellow fever vaccine could be produced by serial passage of virus in chick embryo tissues. Theiler applied this basic approach to develop an experimental attenuated variant of the poliovirus type 2 Lansing strain in 1946 (194). In 1952, Enders, Weller, and Robbins developed the attenuated poliovirus type 1 Brunenders strain by serial passage in cultured cells. Two years later, Li \& Schaeffer developed a highly attenuated derivative of the neurovirulent type 1 Mahoney strain, LSc, by passage at $35^{\circ} \mathrm{C}$ in cultured monkey kidney cells and in monkey skin (104). The LSc strain was further treated by three consecutive single-plaque passages by Albert Sabin (171) to produce the LS-c 2ab strain, generally known as Sabin type 1 (Sabin 1). Sabin also developed attenuated strains for serotypes 2 and 3. The Sabin 2 OPV strain (P712, Ch, 2ab) was derived from virus (P712) that was isolated from a healthy child and shown to have low neurovirulence (171). By contrast, the attenuated Sabin 3 strain (Leon $12 \mathrm{a}_{1} \mathrm{~b}$ ) was produced by rapid passage in monkey kidney cell culture of a highly neurovirulent strain isolated from the spinal cord of a child who had died of bulbo-spinal poliomyelitis (171). In addition to Sabin, two other groups, led by Hilary Koprowski (99) and Herald Cox (17), developed attenuated poliovaccine strains for each serotype. All three sets were carefully evaluated for low neuropathogenicity for monkeys, immunogenicity, genetic stability on human passage, safety (inability to cause paralysis in humans), and restricted capacity to spread (143, 144, 150). The Sabin strains, which had the lowest neuropathogenicity and an excellent safety record from large-scale field trials (170), were approved for worldwide distribution. In the United States, OPV was licensed sequentially (Sabin 1, August 1961; Sabin 2, October 1961; Sabin 3, March 1962). Licensure of Sabin 3 was delayed because of concerns about its undesirable genetic instability and relatively low immunogenicity (149). OPV was initially administered serially in monovalent form, but successful field trials in Canada led to licensure of a trivalent formulation in 1963 (185).

OPV was developed in the 1950 s using the well-established empirical approach of rapid passage of virus at subphysiologic temperatures in cells and tissues of nonhuman origin. Molecular biology was then in its infancy, and although virologists of that period were aware of the rapid advances in bacteriophage genetics (37), they were guided primarily by the principles of genetic selection for spontaneous mutants. OPV development occurred against the backdrop of the disastrous attempts to develop poliovaccines two decades earlier, when investigators failed to distinguish between chemical inactivation of infectivity and attenuation of neurovirulence by genetic selection and were unaware of the existence of multiple poliovirus serotypes (149, 166, 185). However, the rising incidence of 
paralytic polio in developed countries in the early 1950s greatly increased the urgency of developing and deploying effective poliovaccines. It would be another two decades after licensure of OPV that the molecular basis of OPV attenuation became amenable to systematic investigation.

Early attempts to develop improved OPV strains. The genetic stability of OPV was a major concern before $(143,144)$ and after (11) licensure. To minimize the accumulation of neurovirulent revertants in OPV seed stocks, manufacturing processes (permissible cell substrates, incubation temperatures, multiplicities of infection, and the number of passages from the original Sabin seed stocks) for OPV production have been strictly controlled (57). Each production lot is tested for retention of the key phenotypes of low neuropathogenicity for monkeys and temperature sensitivity. These phenotypes are least stable for the Sabin 3 strain (28), which has the highest variability across production lots and is also associated with the highest incidence of VAPP among OPV recipients (see below).

Consequently, several attempts were made to produce a type $3 \mathrm{OPV}$ strain with improved genetic stability. An improved type 3 OPV seed was developed by Pfizer Laboratories in England after extracting the RNA from low-passage Sabin 3 seed, transfecting monkey kidney cells, and screening plaques for low neurovirulence. The new seed stock was more consistent and stable during production and was also free of SV40 contamination (185).

In 1962, Vonka et al. (202) developed a new experimental attenuated type 3 vaccine strain, USOL-D-bac, unrelated to Sabin 3. The new strain appeared to be less neurovirulent and more genetically stable than Sabin 3 (202). The favorable phenotypic properties of USOL-D-bac won approval for field trials testing its efficacy and genetic stability upon administration to children (see below).

Another attenuated type 3 strain, Zhong$\mathrm{III}_{2}$, was developed in China in the 1970 s by classical selection methods (6). The parent to
Zhong- $\mathrm{III}_{2}$, isolated from a healthy unimmunized child, resembled the type 3 wild polioviruses then endemic to China. Subsequent genetic characterization revealed that Zhong$\mathrm{III}_{2}$ was a derivative of Sabin 3, indicating that the healthy infected child had an undetermined exposure to OPV (86) and that a critical IRES determinant of attenuation characteristic of Sabin 3 (see below) had been restored during reattenuation (162).

\section{Genetic Determinants of Attenuation of the Sabin OPV Strains}

Identification of the genetic determinants of attenuation of the Sabin OPV strains has been comprehensively reviewed $(64,126,159,205)$. The first reports of the sequences of complete poliovirus genomes in the early 1980s $(96,158)$ and the development of infectious poliovirus cDNA clones (157) opened the way for systematic investigation of the critical mutations responsible for the attenuated and temperature-sensitive phenotypes of the Sabin OPV strains. A common feature of the Sabin strains is the presence of nucleotide substitutions in the IRES, which in serotypes 1 and 3 are critical attenuating mutations. Additional mutations encoding amino acid substitutions in the capsid region contribute to and stabilize the attenuated phenotype.

Sabin 1. The 57 nucleotide substitutions distinguishing the Sabin 1 strain from its neurovirulent parent, Mahoney, are scattered throughout the genome (138). Six map to the 5'-UTR, 49 map to the coding region (21 of which encode amino acid substitutions), and 2 map to the $3^{\prime}$-UTR. Infectious cDNA constructs containing different combinations of blocks of Sabin 1 and Mahoney sequences were tested for neurovirulence in monkeys or transgenic mice expressing the CD155 receptor, for temperature sensitivity, and for other phenotypic properties distinguishing the two strains $(14,140)$. The single most important determinant of the attenuated phenotype of Sabin 1 was the $A \rightarrow G$ substitution at 
position 480 (abbreviated A480G) in the IRES (83). Four other substitutions contributing to the attenuated phenotype mapped to the capsid region (one in $\mathrm{VP} 4$, one in $\mathrm{VP} 3$, and two in VP1), and one substitution contributing to the temperature-sensitive phenotype (but not to the attenuated phenotype) mapped to the $3 \mathrm{D}^{\mathrm{pol}}$ region $(14,140,148)$ (Figure 2).

The contribution of the remaining substitutions to the attenuated phenotype is difficult to determine. Most of the substitutions in the coding region generate synonymous
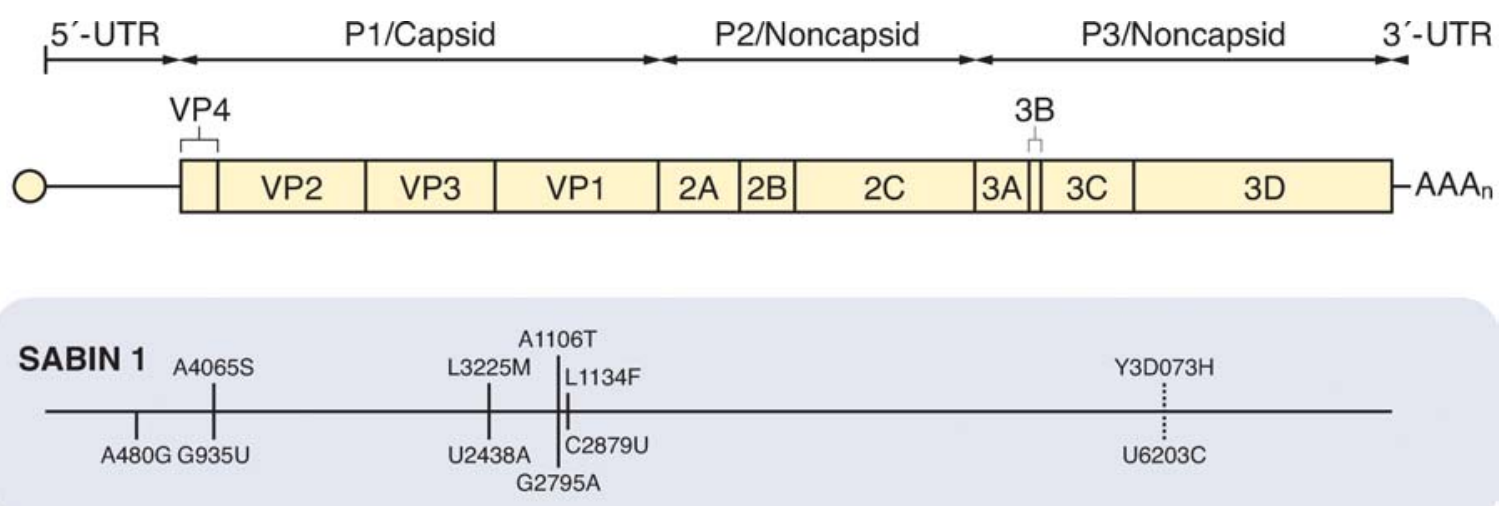

\section{SABIN 2}
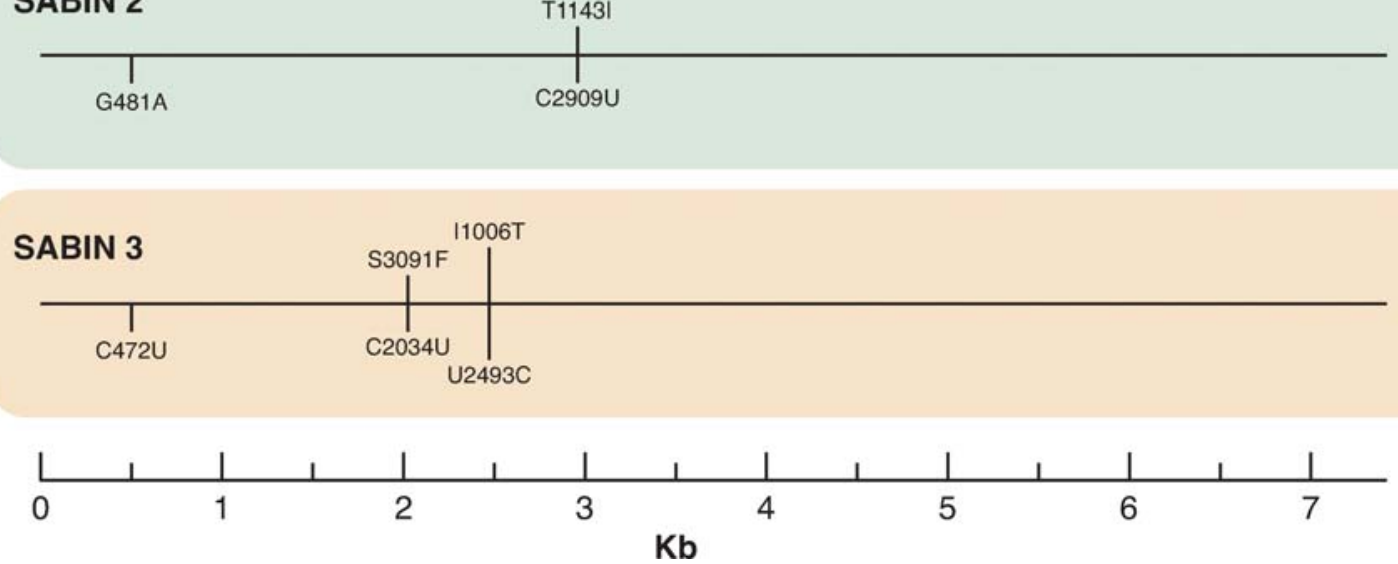

Figure 2

Location of principal attenuating nucleotide (lower bars) and amino acid (upper bars) substitutions in each of the three Sabin OPV strains. Abbreviations of nucleotide residues: A, adenine; C, cytosine; G, guanine; $\mathrm{U}$, uracil. Abbreviations of amino acid residues: A, alanine; C, cysteine; F, phenylalanine; $\mathrm{H}$, histidine; I, isoleucine; L, leucine; $\mathrm{M}$, methionine; S, serine; T, threonine; $\mathrm{Y}$, tyrosine. Substitutions are shown as nonattenuated parent-position-Sabin strain; nucleotide positions are numbered consecutively from residue 1 of the RNA genome; amino acid positions are indicated by the abbreviated name of the viral protein (4, VP4; 2, VP2; 3, VP3; 1, VP1; 3D, 3D-polymerase) and numbered consecutively from residue 1 of each protein. For example, a guanine (Mahoney) $\rightarrow$ uracil (Sabin 1) substitution at RNA position 935 (G935U) encodes an alanine (Mahoney) $\rightarrow$ serine (Sabin 1) replacement at residue 65 of VP4 (A4065S). The Y3D073H substitution in Sabin 1 and S3091F substitution in Sabin 3 are important determinants of temperature sensitivity. Figure summarizes findings from References 14, 83, 111, 160, 191, 203. Redrawn from Reference 185 with permission from Elsevier. 
codons, which may have minimal phenotypic effects, and probably reflect the large number of passages in the attenuation pathway from Mahoney to Sabin 1. Quantitative determination of the contributions of each substitution is complicated by several factors: (a) The role of minor determinants of attenuation is difficult to measure, $(b)$ some substitutions have pleiotropic effects on phenotype, (c) some Sabin strain phenotypes require a combination of substitutions, (d) second-site mutations can suppress the attenuated phenotype in various ways, and (e) the outcome of experimental neurovirulence tests may vary with the choice of experimental animals (monkeys versus transgenic mice) or the route of injection (intraspinal versus intracerebral).

Sabin 2. Only two nucleotide substitutions (G481A in the IRES, and C2909U encoding a $\mathrm{T} \rightarrow \mathrm{I}$ substitution at position 143 of $\mathrm{VP} 1$ ) appear to be responsible for the attenuated phenotype of Sabin $2(111,160)$ (Figure 2). The total number of sequence differences between the parental P712 strain and Sabin 2 is uncertain. However, because P712 has inherently low neurovirulence (171), identification of critical attenuating sites in Sabin 2 involved determination of the effects of introduction of sequences derived from a minimally divergent neurovirulent revertant of Sabin 2 (obtained from a case of VAPP) into infectious cDNA constructs derived from Sabin 2 (111, 160). The precise contribution of the G481A substitution, which is not found in the IRES sequences of type 2 wild polioviruses, to the attenuated phenotype of Sabin 2 is unclear, as variants and even vaccine seed stocks containing the wild-type allele are attenuated in transgenic mice (163).

Sabin 3. Detailed analysis of the attenuated phenotype of Sabin 3 has been possible because the neurovirulent parental strain, Leon, differs from Sabin 3 by only 10 nucleotide substitutions (182). In addition, numerous neurovirulent revertants of the Sabin 3 strain have been isolated from patients with VAPP
(203) and from healthy OPV recipients (109). Only three substitutions (C472U in the IRES, C2034U encoding an $\mathrm{S} \rightarrow \mathrm{F}$ substitution at position 91 of VP3, and U2493C encoding an $\mathrm{I} \rightarrow$ T substitution at position 6 of VP1) appear to be the main determinants of the attenuated phenotype (109, 126, 203) (Figure 2).

In all three Sabin strains, the attenuated phenotype is determined by multiple substitutions. The substitutions in the IRES, which alter stem-loop structures $(63,68,126,132)$ and reduce the efficiency of initiation of translation of the poliovirus RNA template (68, $188)$, contribute most to the attenuated phenotype of the Sabin 1 and Sabin 3 strains. Mutations that restore the original stem-loop structure in the IRES [Sabin 1: G480A (backmutation) or U525C (suppressor); Sabin 2: A481G; Sabin 3: U472C] are frequently found in vaccine-related isolates from healthy OPV recipients $(127,132)$ and patients with VAPP (61), as well as from the environment (228). The role of the IRES to the neurovirulent phenotype of the type 1 Mahoney strain was demonstrated by the reduced neurovirulence of chimeras in which the IRES of Mahoney had been replaced with that of human rhinovirus type 2 (62). In Sabin 3, the critical $\mathrm{C} 472 \mathrm{U}$ substitution reduces the efficiency of binding of the PTB, required for initiation of translation, to the IRES (65). The translational deficit for Sabin 3 is moderate in intestinal cells, where PTB levels are high, but severe in neurons, where PTB levels are low. The precise mechanisms by which the capsid mutations contribute to the attenuated phenotype are less clear. Impairment of the efficiency of binding to the CD155 receptor (14) and reductions in the stability of the capsid (128) may play a role.

Sabin and other developers of OPV strains struck a balance between low neuropathogenicity, good immunogenicity, and acceptable levels of genetic stability (150). The high genetic stability of the Sabin type 1 strain is probably attributable to the greater number of substitutions contributing to the attenuated phenotype. This property is
PTB:

polypyrimidine tract-binding protein 
especially important for the Sabin 1 vaccine strain, because type 1 wild polioviruses typically have high paralytic attack rates and can spread over wide geographic areas in explosive outbreaks $(85,147)$. Sabin 2 may revert more rapidly, but its immunogenicity is high (150, $185)$ and the paralytic attack rates of type 2 wild polioviruses are low (135). Sabin 3 is associated with the highest rates of VAPP (see below), which is probably a result of low genetic stability of the critical attenuating substitution (28), relatively low immunogenicity (184), and an intermediate paralytic attack rate for type 3 polioviruses (135). Nonetheless, all three Sabin strains normally have low pathogenic potentials, and incidence of VAPP in countries with high rates of OPV coverage (183) are $>3000$-fold lower than the incidence of paralytic poliomyelitis in areas with circulating wild polioviruses.

\section{The Remarkable Safety Record of OPV}

In view of the small number of substitutions controlling the attenuated phenotypes of the Sabin strains, it is remarkable that OPV has attained such an outstanding record of safety and efficacy over the four decades of worldwide use $(43,55)$. Most RNA viruses have highly mutable genomes that are potentially capable of rapid evolution, many of orders of magnitude faster than the genomes of DNA viruses or cellular organisms $(41,45)$, and polioviruses are among the most rapidly evolving of all RNA viruses $(60,88,108,117,185$, 226). Moreover, the attenuating mutations of the OPV strains are under strong negative selection when the vaccine replicates in the intestinal tract of OPV recipients $(126,127$, 228 ), and variants with reversions at the key IRES sites are pre-existent in OPV lots (28, 161, 189). Although the selective pressure is probably primarily for increased translational efficiency in intestinal cells (along with loss of temperature sensitivity, increased capsid stability, and possibly more efficient interaction with the CD155 receptor), the revertants (especially type 3 ) excreted by OPV recipients are much more virulent for motor neurons. To counter the daunting challenges of delivering a live, attenuated RNA virus vaccine via its natural route of infection (170), immunization strategies were developed to minimize adverse events $(172,185)$. In developed countries, OPV was first delivered in mass campaigns to achieve high rates of coverage, followed by a strategy of comprehensive routine immunization. Similar strategies were adopted in developing countries, with mass OPV campaigns often playing a more prominent role than routine immunization. In most instances, OPV was delivered in the context of pre-existing high population immunity to poliovirus, because of recent exposure to circulating wild polioviruses or, as with developed countries in the early 1960 s, from the combination of immunity acquired from natural infection and immunity acquired from several years of immunization with IPV. These strategies have likely minimized the epidemiologic consequences of the frequent phenotypic reversion of the OPV strains.

\section{Molecular Approaches Toward the Development of More Stable OPV Strains}

Advances in the understanding of the genetic basis for attenuation of the Sabin OPV strains have generated renewed interest in developing OPV strains with improved genetic stabilities. Nomoto and colleagues $(97,137)$ constructed chimeras with the capsid regions of Sabin 2 and Sabin 3 inserted into the genetic background of the more highly substituted and stable Sabin 1 strain. The same group also developed less neurovirulent strains by introducing deletions into the IRES (81). Agol et al. (1) also constructed attenuated strains by modification of IRES sequences, while Gromeier et al. (62) attenuated Mahoney by substitution of its IRES with the homologous rhinovirus IRES sequences. Finally, Macadam et al. (110) constructed IRES mutants based upon Sabin 3 that had enhanced 
genetic stabilities in vitro. Despite the sophistication of these approaches, the development of attenuated virus vaccines remains highly empirical, and the biological properties of these strains in the field cannot be predicted.

\section{CONTROL OF POLIO BY IMMUNIZATION}

\section{Developed Countries}

The high-income countries of North America, Western Europe, and the southwest Pacific were quick to adopt widespread immunization with IPV. The impact was dramatic. In the United States, for example, the incidence of paralytic polio fell from 13,850 in 1955 to 829 in 1961 (175). Although transition to OPV in the United States was not complete until the mid-1960s, the last peak year for polio was 1963, and all domestic reservoirs for poliovirus circulation apparently had been eliminated before 1970 (94, 164). Similar highly favorable results were achieved in other developed countries $(139,178,201)$.

\section{Developing Countries}

IPV was not widely used in developing countries, and the possibility of widespread immunization against polio had to await the availability of OPV. OPV had several key advantages over IPV for use in developing countries: (a) ease of administration, (b) suitability for mass campaigns, $(c)$ induction of intestinal mucosal (secretory IgA) immunity, and (d) low cost. The synchronous induction of intestinal immunity through mass OPV campaigns efficiently blocks person-to-person transmission of wild poliovirus, thereby protecting both individual vaccine recipients and the wider community. Although the capacity of OPV virus to spread to unimmunized contacts has often been cited as an advantage (170), this benefit is offset by the potential for spread of neurovirulent variants excreted by OPV recipients.
Sabin (172) advocated mass OPV campaigns as the most effective means of polio control. Cuba adopted his approach in 1962 and within a year had stopped all wild poliovirus circulation $(32,120)$. Poliovirus circulation dropped sharply in Brazil after implementation of NIDs in 1980, which was preceded by nearly 20 years of routine OPV immunization that had limited success (165). The dramatic success of mass campaigns in Cuba, Brazil, and Mexico led the PAHO to resolve in 1985 to eradicate polio from the Americas by 1990 (38).

\section{The Global Polio Eradication Initiative}

The PAHO approach of coordinated regional NIDs supplementing improved rates of routine OPV coverage quickly reduced polio incidence. In view of the rapid progress attained in the Americas, the World Health Assembly, which is the governing body for WHO, resolved in 1988 to eradicate polio worldwide by the year 2000 (207), launching the WHO Global PEI. At the time of the World Health Assembly resolution, wild polioviruses were circulating unabated in much of the developing world, with an estimated 350,000 paralytic polio cases occurring in $>125$ endemic countries in 1988 (219) (Figure 3). Since 1988 immunization has spared an estimated 9 million children worldwide from paralytic poliomyelitis. By the end of 2004, poliovirus endemicity was restricted to approximately 10 countries (Figure 3), and the total number of polio cases worldwide was estimated from comprehensive surveillance to be 1253 , a reduction of $>99 \%$ in 16 years. The impact of the global initiative can be monitored by the declining genetic diversity of wild poliovirus genotypes and lineages (Figure 4). Wild poliovirus type 2 has probably been eradicated, as it was last detected in October 1999 in Uttar Pradesh, India (208). Wild poliovirus type 3 survives by steadily diminishing chains of transmission, and in 2004 it was endemic to parts of only five countries (Nigeria, Niger,
NIDs: National Immunization Days

PAHO: Pan American Health Organization

PEI: Polio

Eradication Initiative 
SNIDs: Subnational Immunization Days
Pakistan, India, and Sudan) (Figure 5). Wild poliovirus type 1 is highly localized in India, Pakistan, Afghanistan, and Egypt, but it remains more widespread in West and Central Africa following outbreaks in 2003-2004 of virus originating from northern Nigeria $(218,222)$ (Figure 5). Three WHO regions have been certified free of indigenous wild poliovirus circulation, the Americas in 1994 (last indigenous case: Peru, 1991) (19), the Western Pacific Region in 2000 (last indigenous case: Cambodia, 1997) (22), and the European Region in 2002 (last indigenous case: Turkey, 1998) (23) (Figure 3). Regular updates on the progress toward global polio eradication are posted on the WHO website (http://www.polioeradica tion.org/).

Biological principles of poliovirus eradication. The key biological requirements for poliovirus eradication are $(a)$ absence of a persistent carrier state, (b) virus spread is by person-to-person transmission, $(c)$ immunization interrupts virus transmission, $(d)$ absence of any nonhuman reservoir hosts for the virus, and (e) finite virus survival time in the environment (42). An additional important nonbiological requirement for any disease eradication effort is political will, arising from the perceived benefits of eradication, and expressed internationally through resolutions passed by the World Health Assembly. Essential to the success of the Global PEI has been the strong alliance among national governments, international agencies such as UNICEF, and private partners, including Rotary International (whose international PolioPlus campaign began in 1979) (114, 176), the UN Foundation, and the Bill and Melinda Gates Foundation.

Basic strategy for polio eradication. The basic strategy of the PEI is (a) high routine immunization coverage of infants with OPV, (b) supplementary OPV immunization through NIDs and SNIDs, (c) targeted doorto-door "mop-up" OPV immunization in areas of focal transmission, and (d) sensitive surveillance for poliovirus (80). High rates of routine OPV immunization are required to block poliovirus circulation in areas where the risk factors converge, conditions under which routine OPV coverage rates exceeding $90 \%$ may be insufficient to block poliovirus circulation $(55,147,165,185)$. Such rates are currently unattainable through routine immunization in the least developed countries. Supplementary immunization is the mainstay of polio eradication in developing countries, and has been instrumental in raising population immunity rates above the thresholds required to block poliovirus transmission (210). Supplementary immunization strategies are driven by poliovirus surveillance, which is used to guide the intensified SNIDs and mop-up campaigns to the reservoir communities where the chains of poliovirus transmission continue to survive and propagate.

Figure 3

Geographic distribution of polio-endemic and polio-epidemic countries in 1988, 1998, and 2004.

Polio-endemic countries are colored red, nonendemic countries are colored yellow, and countries within WHO regions certified as polio-free are colored blue. Countries experiencing repeated cross-border importation of wild poliovirus (Iran and Myanmar in 1998) are indicated by a red-to-yellow gradient, countries with re-established transmission of more than 1 year (2004 map) are indicated with red and yellow hatch pattern, countries with sporadic importation within a single year (2004 map) are indicated by red shading over yellow background, and countries with single cases associated with imported wild poliovirus (Canada in 1988; Botswana and Ethiopia in 2004) are shown with a red dot at the location of each case. The two red dots in Saudi Arabia (2004) represent two cases associated with separate importations of wild poliovirus from epidemic areas in Sudan. Updated from Reference 87 with permission from the American Society for Microbiology. 

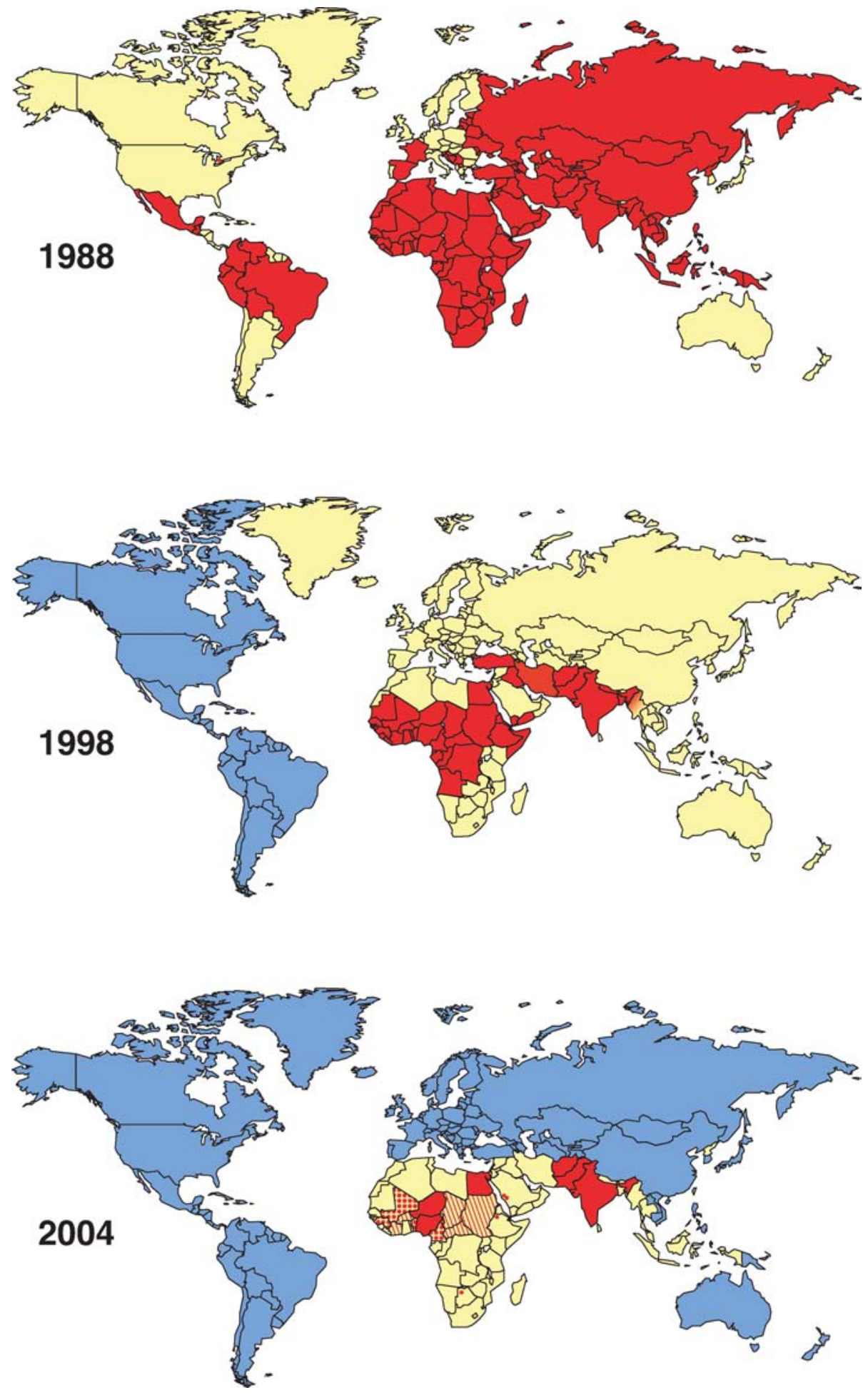
South Asia (SOAS)

Northeast Africa (NEAF)
West Africa-B (WEAF-B): endemic

West Africa-B (WEAF-B): re-established

West Africa-B (WEAF-B): sporadic
Figure 4

Progressive eradication of wild poliovirus genotypes, 1988 to 2004. Stop signs indicate the year and location where the last isolate was obtained for each extinct genotype. Surviving type 1 and type 3 poliovirus genotypes are colored-coded; hatch and shading patterns indicate, respectively, countries with re-established transmission and sporadic importation of West Africa genotype viruses. Redrawn from Reference 87 with permission from the American Society for Microbiology.
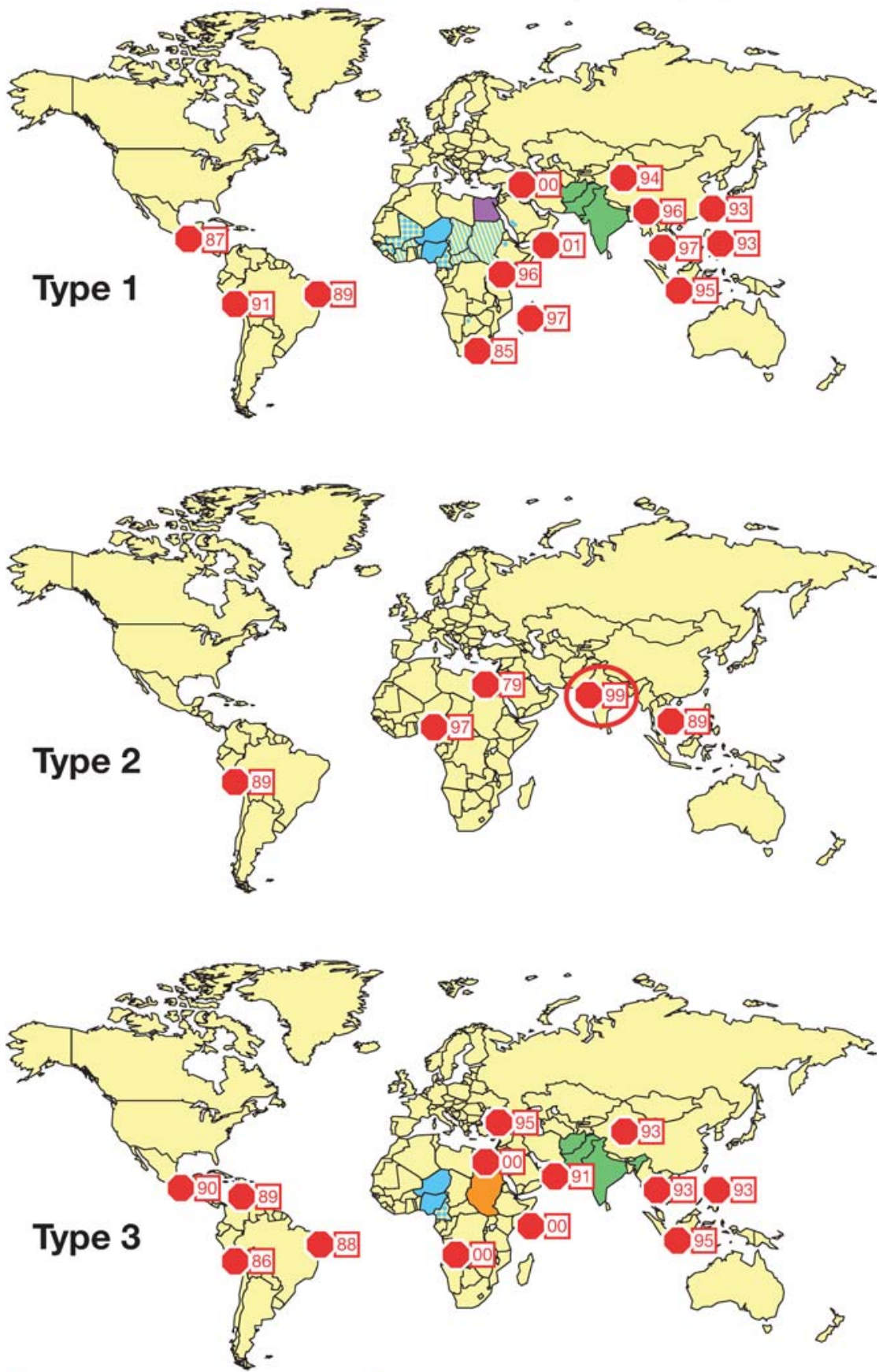

South Asia (SOAS)

Central Africa (CEAF)
West Africa (WEAF): endemic (1) West Africa (WEAF): sporadic 
AFP and poliovirus surveillance. Surveillance for wild polioviruses has two arms: (a) AFP case investigations and (b) virologic studies of polioviruses obtained from clinical specimens or other sources such as the environment. Because AFP has multiple etiologies [including Guillian-Barré syndrome, transverse myelitis, and transient (or occasionally permanent) paralyses associated with NPEV infections] $(4,55,142,185)$, AFP surveillance must be combined with virologic studies to investigate the possible etiologic role of wild polioviruses.

AFP surveillance is also limited in sensitivity, because only about 1 in 200 nonimmune children infected with wild poliovirus shows signs of AFP $(4,55,142)$. In populations with higher levels of immunity, AFP cases may appear in fewer than 1 of 10,000 wild poliovirus infections (155). Nonetheless, over time all effectively performing AFP surveillance systems can detect endemic poliovirus circulation. In suspected high-risk areas lacking effective AFP surveillance, supplementary surveillance activities, such as sampling community contacts of AFP cases, stool surveys of healthy children, or environmental sampling, have been implemented to increase sensitivity for detecting wild polioviruses $(39,48,116$, 131, 155, 190, 197).

Poliovirus surveillance methods. The virologic tools of poliovirus surveillance are well developed. A global network of 145 formally accredited and highly competent poliovirus laboratories has been established by WHO to apply these tools in support of polio eradication $(209,214,216)$. Standard methods for poliovirus isolation in cultured cells (217) have been enhanced by the use of recombinant murine cells expressing the CD155 receptor $(79,151)$. Polioviruses can be distinguished from NPEVs either by using standard typing assays (217) or by the PCR using poliovirus group-specific (93) or serotypespecific (92) primer sets. ITD of poliovirus isolates (testing whether they are vaccine related or wild) is performed throughout the global network using one antigenic and one molecular method (21). The standard antigenic ITD method uses an ELISA system with preparations of highly specific cross-adsorbed antisera $(198,199)$. The molecular ITD methods use genotype-specific nucleic acid probes $(35,36)$, genotype-specific PCR primers (105, 224, 225), or PCR-RFLP (9).

ITD screens for wild polioviruses and VDPVs and screens out "OPV-like" polioviruses that are unlikely to be of current epidemiologic importance. Since 2001 network laboratories routinely sequence the complete VP1 region ( $\sim 900 \mathrm{nt}, \sim 15 \%$ of the total genome; Figure 1) of all wild poliovirus and VDPV isolates. VP1 sequences are used for routine comparisons because they encode several serotype-specific antigenic sites (124) (Figure 1) and evolve primarily by successive fixation of nucleotide substitutions. Because of the exceptionally rapid rate of poliovirus sequence evolution (equivalent to 1 to 2 nucleotide substitutions per week over the entire genome; see below), comparative VP1 sequence data generally afford sufficient phylogenetic resolution for reconstruction of individual chains of transmission and identification of local endemic reservoirs (179), but sequencing windows may be widened to cover the complete poliovirus genome if higher resolution is needed $(84,108,177,223)$. Sequence relationships are generally summarized in the form of phylogenetic trees and lineage maps $(10,12,48,84,87,118,177$, 179, 226).

Rapid poliovirus evolution and poliovirus surveillance. The rapid evolution of polioviruses during natural replication permits the patterns of poliovirus transmission to be followed with unparalleled precision $(108,179)$. Nucleotide substitutions (>80\% of which in the coding region are to synonymous codons) accumulate at an overall rate of $\sim 1 \%$ per year at all sites, and at $\sim 3 \%$ per year at synonymous sites $(10,60$, $85,88,108,117)$. Evolution rates appear to be similar across serotypes and between wild
AFP: acute flaccid paralysis

NPEV: nonpolio enterovirus

PCR: polymerase chain reaction

ITD: intratypic differentiation

RFLP: restriction fragment length polymorphism 

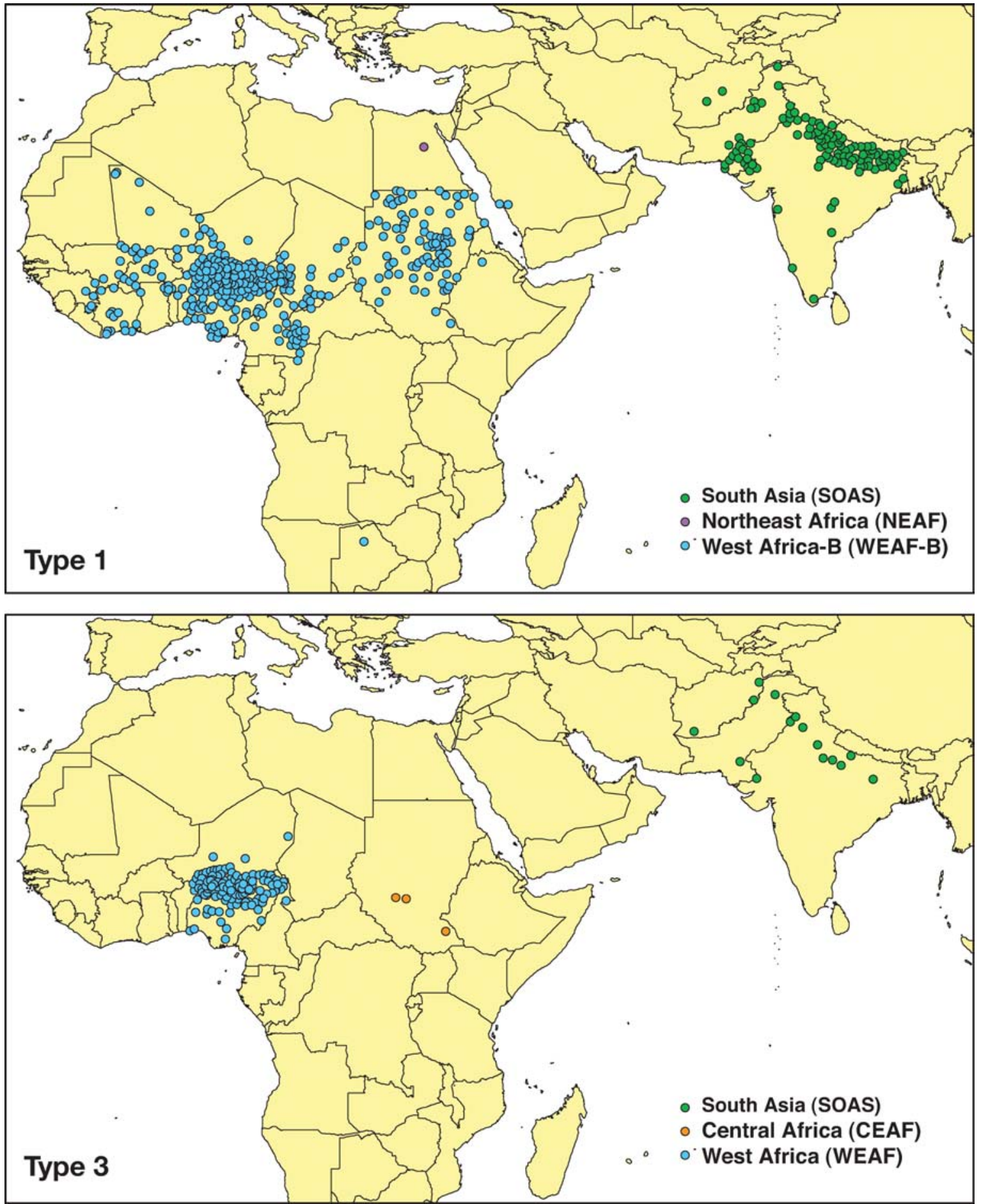
polioviruses and VDPVs $(60,84,117,185$, $223,226)$. Interestingly, the bottlenecks driving the rapid evolution of polioviruses appear to occur primarily during replication in the human intestine $(95,109)$. They also appear to be largely independent of immune selection, as evolution rates are similar during prolonged replication in immunodeficient patients $(10,88,117,223)$ and during widespread circulation $(60,84,85,108)$.

Polioviruses actively recombine with each other $(18,30,33,59,91,106,223)$ and with the closely related species $\mathrm{C}$ human enteroviruses $(15,66,84,107,169,177,226)$. The nature and location of recombination junctions provide additional markers for the resolution of poliovirus lineages $(84,107)$, especially for closely related isolates (27). The dynamic evolution of wild polioviruses appears to have few biological consequences, as cocirculating genotypes and recombinant lineages of the same genotype are frequently found in endemic areas (107), and the biological properties of wild polioviruses within each serotype appear to be similar. On the other hand, the dynamic evolution of vaccine viruses is the underlying cause of adverse events associated with the use of OPV.

\section{VACCINE-ASSOCIATED PARALYTIC POLIOMYELITIS}

After more than 40 years of use and many billions of doses distributed worldwide, OPV has been associated with very few adverse events. The most commonly recognized adverse event is VAPP, which is clinically indistinguishable from polio caused by wild polioviruses. The first cases of VAPP were recognized within a year of licensure of OPV, and all of the early cases were associated with the Sabin 3 strain (193). The Sabin 3 association was unambiguous because OPV had been delivered in monovalent form (185). VAPP rates are low and similar in most countries (5, $51,82,98,185,204)$. In the United States, the risk of VAPP in first-dose OPV recipients is about 1 case per 750,000 children immunized (20). The risk of VAPP decreases sharply ( $>10$-fold) for subsequent doses. VAPP cases are sporadic and occur in both OPV recipients and their unimmunized household and nonhousehold contacts. A small proportion ( $7 \%$ ) of VAPP cases in the United States are classified as "community-acquired," indicating no known exposure to OPV. VAPP in OPV recipients and household contacts is most frequently associated with Sabin 3 (71\% of cases), followed by Sabin 2 (26\% of cases) (183). VAPP in nonhousehold contacts and in community-acquired cases is most frequently associated with Sabin 2 (50\% of cases), followed by Sabin 3 (33\% of cases) (183). Sabin 1 is rarely associated with VAPP in immunocompetent individuals $(51,183)$.

Poliovirus isolates from immunocompetent VAPP cases show only limited genetic divergence from the parental OPV strains, although the key substitutions conferring the attenuated phenotype have frequently reverted $(61,127)$. Many isolates from VAPP cases, AFP cases with incidental isolation of vaccine-related virus (much more frequent than VAPP cases), and healthy OPV recipients are vaccine/vaccine recombinants $(18,33,59$, $91,106)$. The biological and genetic properties of viruses isolated from healthy OPV recipients/contacts are often indistinguishable

Figure 5

Distribution of polio cases associated with wild polioviruses in 2004. Major wild poliovirus genotypes for each serotype (upper panel, type 1, 1042 cases; lower panel, type 3, 217 cases) are color-coded. In 2004, wild poliovirus circulation became increasingly localized in India, Pakistan, and Afghanistan; remained highly focal in Egypt; and became more widespread in West and Central Africa. Map prepared by Paul Chenoweth from surveillance data provided by the WHO

(http://www.polioeradication.org/content/fixed/casemap.shtml) and the WHO Global Polio Laboratory Network as of 16 February 2005. 
from viruses isolated from patients with VAPP $(125,127)$.

Persons with primary B-cell immun-

iVAPP:

immunodeficiencyassociated

VAPP

iVDPVs:

immunodeficient

VDPVs

cVDPVs: circulating VDPVs

aVDPVs: ambiguous VDPVs odeficiencies (see below) (168) should not be given OPV because they are at a much higher ( 3000-fold) risk for $\operatorname{VAPP}(90,186)$. However, some children have received OPV before their immunodeficiency was recognized. iVAPP differs markedly from VAPP in immunocompetent individuals, as it is rarely associated with Sabin 3 (14\% of cases) and is more frequently associated with Sabin 1 (31\% of cases) and Sabin 2 (72\% of cases) (some patients were infected with more than one serotype) (90). On the other hand, persons with T-cell immunodeficiencies, including those infected with HIV, do not appear to be at an elevated risk for VAPP $(74,90,183$, 186).

Each case of VAPP is an independent event. It is estimated that 250 to 500 cases of VAPP occur worldwide, mostly in countries free of circulating wild poliovirus $(184,220)$. VAPP is a direct clinical consequence of the genetic instability of the Sabin OPV strains, and the most effective means to prevent VAPP is to stop OPV use $(3,220)$.

\section{VACCINE-DERIVED POLIOVIRUSES}

\section{Categories of VDPVs}

Three categories of poliovirus isolates are recognized: (a) OPV-like isolates, which differ from the respective parental Sabin strains by $<1 \%$ of VP1 nucleotides; (b) VDPVs, which differ from the parental Sabin strains at $1 \%$ to $15 \%$ of VP1 nucleotides (i.e., having $\geq 10$ nucleotide substitutions); and (c) wild polioviruses, which differ from the Sabin strains at $>15 \%$ of VP1 nucleotides (209). VDPVs, in turn, are divided into three categories: (a) iVDPVs isolated from immunodeficient patients who have prolonged infections after exposure to OPV (Table 1); (b) cVDPVs that are associated with sustained person-to-person transmis- sion (Table 2); and (c) aVDPVs, clinical isolates from patients with no recognized immunodeficiency and not associated with an outbreak, or environmental isolates whose ultimate source has not been identified.

The demarcation between OPV-like isolates and VDPVs of $>1 \%$ VP1 divergence implies that replication of vaccine virus, either within an individual and during personto-person transmission, had occurred for approximately 1 year or more, in contrast to the normal period of poliovirus excretion of 4 to 8 weeks (2). The definition is based upon the likelihood that VDPVs have had a history of prolonged replication since administration of the initiating OPV dose, but it makes no inference about the biological properties of VDPVs. It does not imply that isolates having $<1 \%$ divergence would lack the capacity to cause paralytic disease in humans or be unable to initiate sustained person-to-person transmission in poorly immunized populations. Indeed, the critical attenuating mutations of the Sabin strains frequently revert well before nucleotide substitutions accumulate to the level of $1 \%(126,127,228)$.

The $1 \%$ definition, while serving as a simple guideline for poliovirus surveillance, is also firmly grounded on observation. Most vaccine-related poliovirus isolates have diverged from the parental vaccine strain by $<0.5 \%$ (34). Although one or two VP1 amino acid residues are selected against during replication of the Sabin strains in the intestine $(58,154)$, it is primarily synonymous substitutions that accumulate over time. Under the assumption that the rate of fixation of synonymous substitutions is approximated by a Poisson model, the $95 \%$ confidence interval of the estimated duration of replication for an isolate with 10 VP1 substitutions would be from 6 months (still prolonged) to 22 months. On the other hand, viruses that had been replicating for a year may have as few as 4 and as many as 16 substitutions (within the predicted $95 \%$ confidence interval; the actual numbers would likely be slightly higher because of substitutions encoding selected amino acid 


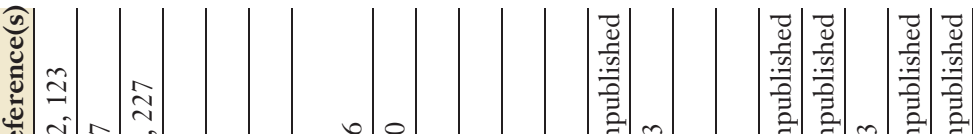

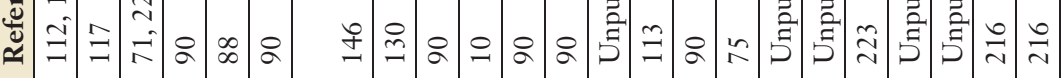

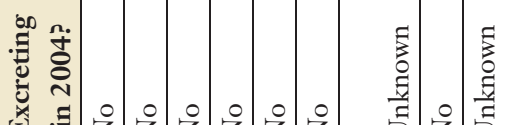

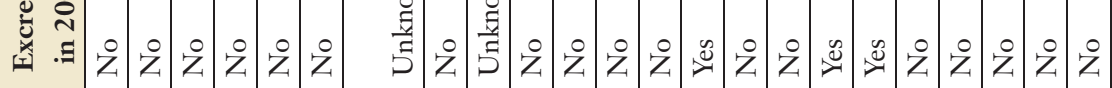

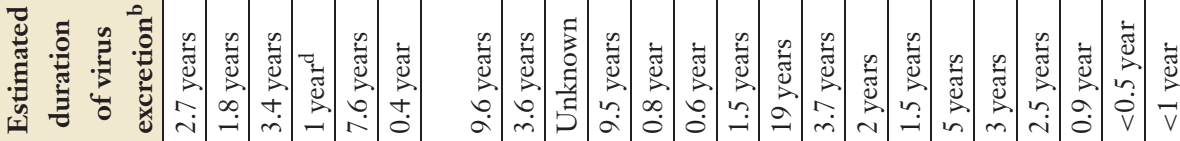

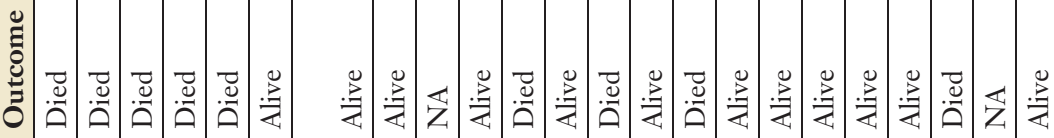

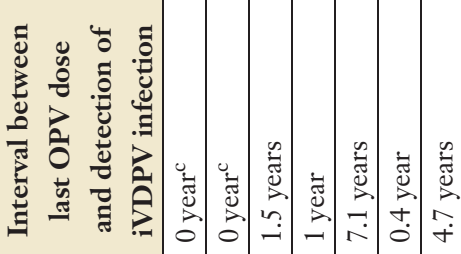

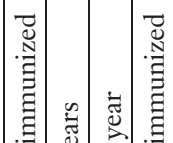

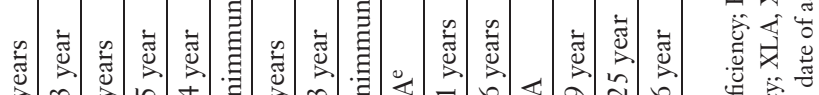

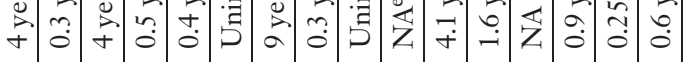

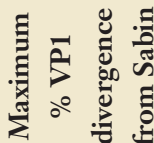
言 吾

i

童

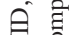

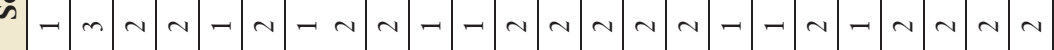

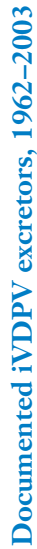

竞

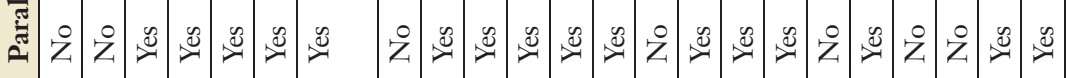

O)

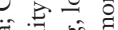

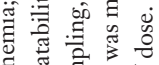

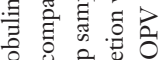

응

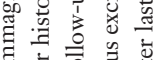

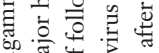

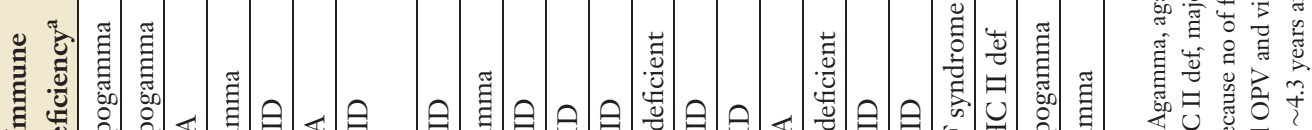

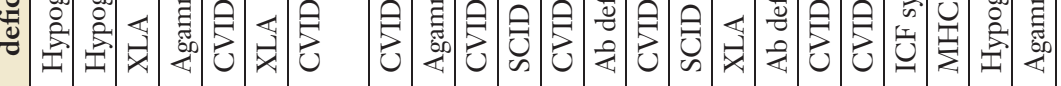

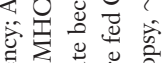

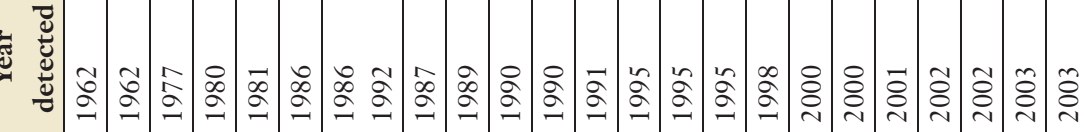

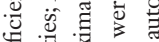

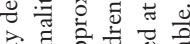

굴

至

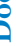

(1)

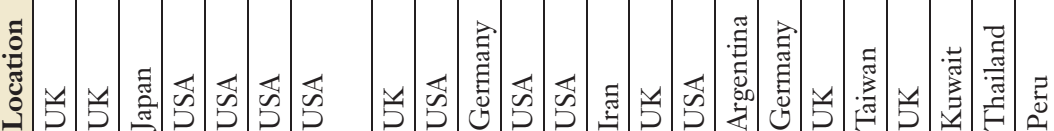
(1) III (4)

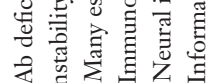


Table 2 Outbreaks of cVDPVs, 1988-2004

\begin{tabular}{|c|c|c|c|c|c|c|c|}
\hline Location & Years & $\begin{array}{c}\text { Reported } \\
\text { number of } \\
\text { polio cases } \\
\end{array}$ & Serotype & $\begin{array}{c}\text { Number of } \\
\text { isolates (\% VP1 } \\
\text { divergence) }\end{array}$ & $\begin{array}{l}\text { Recombination } \\
\text { with Species C } \\
\text { enteroviruses }^{\mathrm{a}}\end{array}$ & $\begin{array}{l}\text { Estimated } \\
\text { duration of } \\
\text { circulation }\end{array}$ & Reference \\
\hline Hispaniolab $^{\mathrm{b}}$ & 2000-2001 & 21 & 1 & $31(1.9-2.6)$ & Yes & 2.5 years & 84 \\
\hline Philippines & 2001 & 3 & 1 & $4(3.1-3.5)$ & Yes & 2.5 years & 177 \\
\hline China $^{\mathrm{d}}$ & 2004 & 2 & 1 & $4(1.0-1.2)$ & No & 1 year & 216 \\
\hline
\end{tabular}

${ }^{a} \mathrm{All}$ cVDPV isolates except those from China were vaccine/nonvaccine recombinants. Multiple distinct cVDPV recombinants were detected in Hispaniola (4 recombinant classes) and Egypt (22 recombinant classes).

${ }^{\mathrm{b}}$ The Hispaniola outbreak includes 13 reported cases from Haiti and 8 cases from the Dominican Republic.

${ }^{\mathrm{c}}$ An unrelated type $2 \mathrm{aVDPV}$ isolate (1\% VP1 divergence) was obtained from an AFP case in a separate community in Madagascar in 2001.

${ }^{\mathrm{d}}$ VDPVs were isolated from 4 polio patients (March and April 2002) and from 2 healthy children (from a stool survey of 316 healthy children conducted in June 2002 in the outbreak area) (M. Rakoto-Andrianarivelo, personal communication).

${ }^{\mathrm{e}}$ Localized outbreak in Guizhou Province.

CVID: common variable immunodeficiency replacements). Thus, the $1 \%$ definition for VDPVs would exclude nearly all isolates that had been replicating for less than 6 months while including most isolates that had a history of replication for at least one year (209, 214). Moreover, cVDPV isolates having $>1 \%$ VP1 sequence divergence probably had circulated for at least one year, with transmission continuing through at least one low transmission season.

It should be emphasized that VAPP is a clinical condition, whereas VDPVs are viruses with unusual genetic properties. VAPP cases have documented temporal association between receipt or exposure to OPV and onset of paralysis (183). VDPV isolates demonstrate higher genetic divergence from OPV strains than do isolates from most VAPP cases, implying an exceptional clinical or epidemiologic history for VDPVs.

\section{iVDPVs from Long-Term Poliovirus Excretors}

A small proportion of individuals with Bcell immunodeficiencies exposed to OPV develop iVAPP (69). A much smaller proportion ( $16 \%$ ) of iVAPP patients become long-term excretors of iVDPVs (90). To date, 23 longterm iVDPV excretors have been identified worldwide by WHO since 1962 (Table 1). Although most of the iVDPV excretors had paralysis, a substantial proportion $(30 \%)$ has shown no signs of paralysis. More than half (57\%) of the patients for which the outcome is known have survived, and most survivors have spontaneously stopped iVDPV excretion. Deaths are usually attributable to VAPP or other complications of immunodeficiency (90).

Prolonged iVDPV excretion among patients with iVAPP is associated with several different primary immunodeficiencies, including CVID, hypogammaglobulinemia, agammaglobulinemia (including $\mathrm{X}$-linked agammaglobulinemia), severe combined immunodeficiency, "antibody deficiency," and major histocompatability complex class II molecule deficiency (Table 1). Patients with CVID have the most prolonged periods of iVDPV excretion and the most divergent iVDPV isolates. Although some patients with CVID have spontaneously stopped virus excretion, at least four have shed virus for more than five years. Three of the recognized prolonged iVDPV excretors with CVID had no signs of paralysis (Table 1). Other CVID patients developed paralysis several years after receipt of the implicated OPV dose (typically the last dose) $(10,88,223)$. One CVID 
patient from the United States was concurrently infected with highly evolved iVDPVs of serotypes 1 and 2. iVDPV isolates are most frequently of types $1(33 \%)$ and $2(62 \%)$, and only rarely of type 3 (4\%) (Table 1).

CVID is the most prevalent $(\sim 1$ in 50,000) of the primary immunodeficiency disorders (168). Symptoms of immunodeficiency typically become evident later in life, usually in the second and third decades, even though prolonged poliovirus infections may have begun years before the diagnosis of CVID $(10,88$, 223). Close relatives of CVID patients have a much increased incidence of IgA deficiency (168), which is also associated with longer times of poliovirus excretion, although excretion rarely persists beyond 6 months (174).

In upper-income and middle-income countries, survival of patients with primary immunodeficiency disorders is extended by treatment with intravenous immunoglobulin, allowing many patients to lead normal lives $(69,168)$. All of the patients known to be longterm poliovirus excretors have been from upper-income and middle-income countries (Table 1). Persons with primary immunodeficiency disorders are unlikely to survive in low-income countries (or in low-income communities in many middle-income countries) because of lack of access to supportive therapy (69).

\section{cVDPVs from Polio Outbreaks}

Hispaniola, 2000-2001. The first cVDPV outbreak to be detected occurred in 20002001 on the Caribbean island of Hispaniola, divided between Haiti and the Dominican Republic (84). Two AFP cases with onset in mid-summer of 2000, one from the Dominican Republic and the other from Haiti, were associated with poliovirus type 1 . Because type 1 is the serotype most frequently associated with polio outbreaks (147), and because the cases occurred during the seasonal peak for wild poliovirus circulation, it was initially suspected that the cases were associated with imported wild poliovirus. However, the isolates from the two cases were unrelated ( $<82 \%$ VP1 sequence identity) to any known wild polioviruses, but closely related ( $~ 98 \%$ VP1 sequence identity) to the Sabin $1 \mathrm{OPV}$ strain. Moreover, they shared numerous nucleotide differences from Sabin 1, suggesting that they were derived from a common genetic variant of the vaccine strain, an observation consistent with virus circulation. PAHO was notified that an outbreak involving circulating vaccine virus was suspected, and active search for additional AFP cases was immediately launched. Twenty-one confirmed poliomyelitis cases (13 in the Dominican Republic in 2000, and 8 in Haiti in 2000-2001 including 2 fatal cases) were detected, and 31 cVDPV isolates were obtained from polio patients and their contacts (Table 2) (Figure 6). The patterns of cVDPV transmission were reconstructed from the sequence relationships among the isolates, and a single OPV dose given in late 1998 or early 1999 was implicated as the source of the outbreak (84). The close sequence relationships among isolates from the Dominican Republic suggested that the outbreak there began with the importation of cVDPV from Haiti in the spring of 2000. By contrast, the Haitian isolates were more diverse and appeared to have diverged into at least four separate lineages in 1999. The outbreak stopped in both countries after mass administration of OPV on NIDs (84).

Circulation of VDPV occurred in an environment of low OPV coverage throughout Haiti ( $<30 \%$ nationwide, and as low as $7 \%$ in some areas) and in the affected communities of the Dominican Republic (20\%-30\%). All but one of the patients were either unvaccinated or incompletely vaccinated children. No mass OPV immunization campaigns in the form of NIDs had been conducted in either country within the preceding five years.

Philippines, 2001. The Hispaniola outbreak showed conclusively that low OPV coverage carried a risk of cVDPV emergence and prompted a reassessment by WHO of the strategies both for polio immunization and 


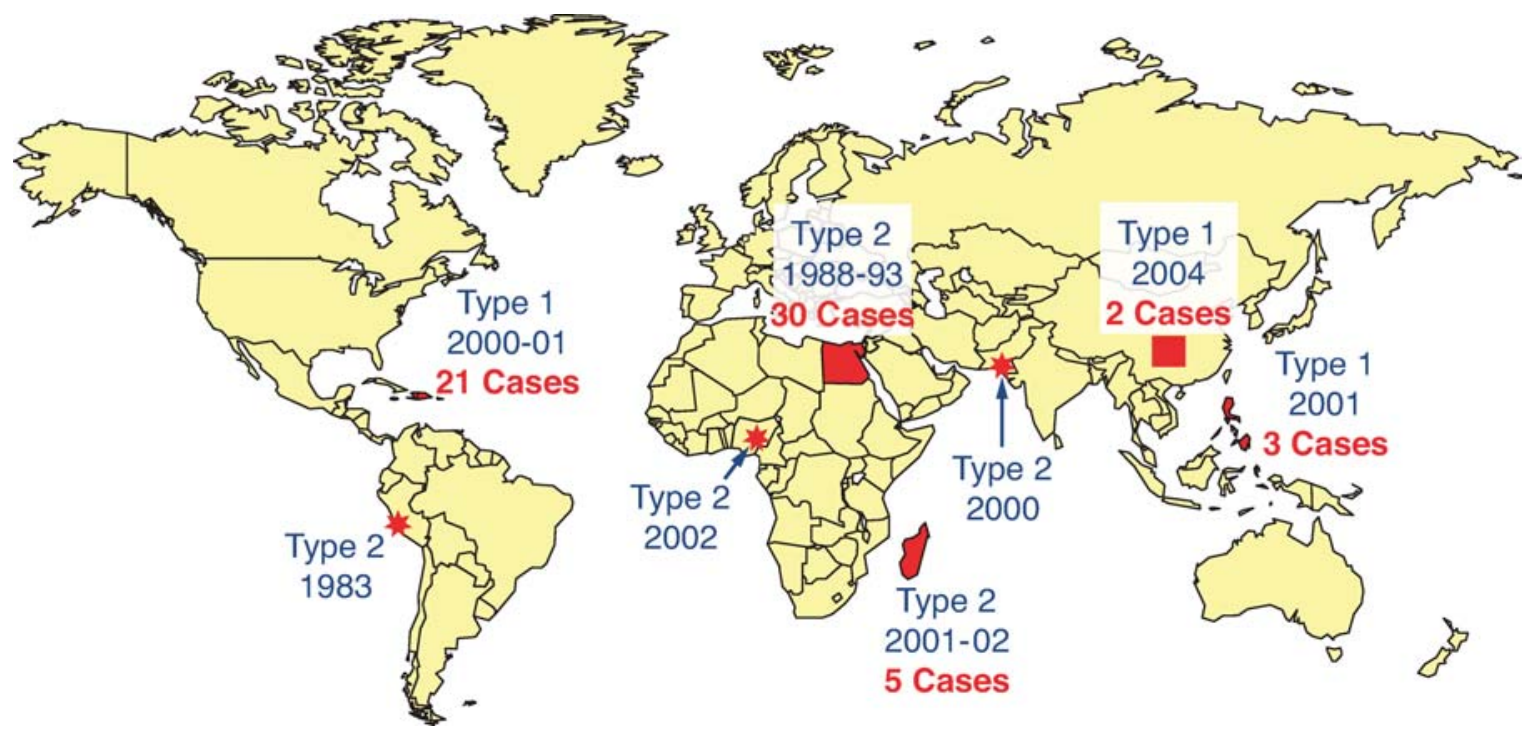

Figure 6

Location of the five polio outbreaks associated with cVDPVs. Shown are the serotypes of the cVDPV isolates, the years of cVDPV virus isolation, and the number of reported polio cases associated with cVDPVs. Locations of single aVDPV isolates genetically similar to cVDPVs and found in areas with low poliovaccine coverage are indicated by asterisks. Redrawn from Reference 89 with permission from the World Health Organization.

poliovirus surveillance. In 2001, the WHO Global Polio Laboratory Network implemented additional testing requirements (see below) for all polioviruses under investigation to increase sensitivity for detecting VDPVs (209). Soon afterwards, cVDPV was detected in the Philippines (177). Specimens from three cases of AFP, reported from March to July 2001, were positive for type $1 \mathrm{cVDPV}$. The isolates were closely related to the Sabin 1 OPV strain ( 97\% VP1 sequence identity) but even more so to each other ( $>99 \%$ VP1 sequence identity) (Table 2). The VP1 sequence relationships among the isolates suggested that the VDPV circulation began with an OPV dose given in 1998. Although the three cases occurred in separate communities (two in Luzon, one in Mindanao), the close sequence similarities among the CVDPV isolates suggested that the virus had spread via a single, minimally branched chain, in contrast to the pattern of multichain transmission found in Hispaniola.
Madagascar, 2001-2002. Four cases of AFP (onsets in March to April 2002) associated with a type $2 \mathrm{cVDPV}$ were clustered in a rural village in the southern Toliara Province of Madagascar (169). These cases were preceded by a case (onset in October 2001) associated with a type 2 aVDPV from the urban district of Toliara, $\sim 400 \mathrm{~km}$ from the rural village. None of the patients had been fully immunized against polio. The type 2 polioviruses isolated from patients and contacts from the two areas represented two geographically separate, genetically distinct, independent VDPV lineages (169). The rural cVDPV isolates differed from Sabin 2 at $\sim 2.5 \%$ of VP1 nucleotides, and the urban aVDPV isolates differed from the Sabin 2 OPV strain at $1 \%$ of VP1 nucleotides (Table 2).

Guizhou, China, 2004. China has maintained sensitive surveillance for wild polioviruses since the early 1990s, and for VDPVs since 2001. In the summer of 2004, type 
$1 \mathrm{cVDPV}$ was isolated from two patients with AFP and from two contacts from a village in Guizhou Province in southern China (W. $\mathrm{Xu}$, personal communication). Neither the patients nor their contacts had received OPV, and overall OPV coverage in the area had declined in recent years. The four isolates differed from Sabin 1 at 9 to $11(1.0 \%-1.2 \%)$ VP1 positions and all four isolates shared identical nucleotide substitutions at 5 positions. Mop-up campaigns were conducted in late summer, and no additional cVDPV isolates have been detected.

\section{Evidence of Past VDPV Circulation}

Egypt, 1983-1993. From 1988 to 1993, 30 cases of poliomyelitis associated with type 2 cVDPV were found in seven governorates of Egypt. The cases occurred at a time of low OPV coverage, and after the apparent eradication of the type 2 wild poliovirus indigenous to Egypt (last known isolate was from a 1979 case) (226). The type 2 isolates were originally thought to be wild polioviruses, but recent molecular studies have shown them to be cVDPVs (226). The sequence properties of the isolates suggested that VDPV circulation in Egypt started with an OPV dose given in 1983 and that progeny from the initiating infection circulated for approximately a decade within Egypt. Like wild polioviruses, the type $2 \mathrm{cVDPVs}$ established independent reservoirs of endemicity within the country. VDPV circulation stopped with rising OPV coverage, and VDPVs were last detected in Egypt in 1993.

Poland, 1968. In 1968, a large poliomyelitis outbreak (464 cases) in Poland immediately followed a field trial of the experimental type 3 oral poliovaccine strain, USOL-D-bac (101). The outbreak occurred in an environment of low population immunity to poliovirus type 3 , because the Sabin 3 component was never included in OPV (owing to difficulties in producing consistent Sabin 3 lots), and the replacement type 3 IPV failed to induce high levels of immunity (101). Although the epidemiologic findings implicated a breach in quarantine of USOL-D-bac recipients during the field trial (101), conclusive evidence for the vaccine origin of the outbreak came from retrospective oligonucleotide fingerprinting (86) and nucleotide sequencing (118) studies of outbreak isolates. Because the maximum extent of VP1 divergence among the available outbreak isolates was $<1 \%$, the isolates do not meet the strict definition for VDPVs; however, the combined epidemiologic (101) and virologic (118) findings show that derivatives of the USOL-D-bac vaccine strain had spread extensively by person-to-person transmission for $\sim 13$ months. Although the implicated vaccine virus was genetically distinct from Sabin 3 (118), the Poland outbreak is the only one known to be associated with any type 3 vaccine strain.

\section{Other Possible Examples of VDPV Circulation}

In a recent retrospective study, evidence was found for the circulation type 2 vaccinerelated virus in Byelorussia (Belarus) following local cessation of OPV use from 1963 to $1966(100)$. In the months after the limited reintroduction of OPV in 1965, type 2 vaccine-related poliovirus was isolated from nine healthy unvaccinated children. The sequence properties of three of the four available isolates showed evidence of prolonged vaccine virus replication (6 to 9 months) (100) (Table 3). In Romania in 1980, a type 1 VDPV was isolated from a patient with "community acquired" VAPP (61). The patient was immunocompetent but lived in a community with low rates of OPV coverage and poor sanitation. In Russia in 1999, an immunocompetent seven-month-old orphanage child contracted VAPP associated with a type 1 VDPV (26). In Romania in July 2002, a type 1 VDPV was isolated from one AFP case (an unimmunized five-month-old child) and seven healthy unimmunized children within a Roma community with low rates of OPV coverage 


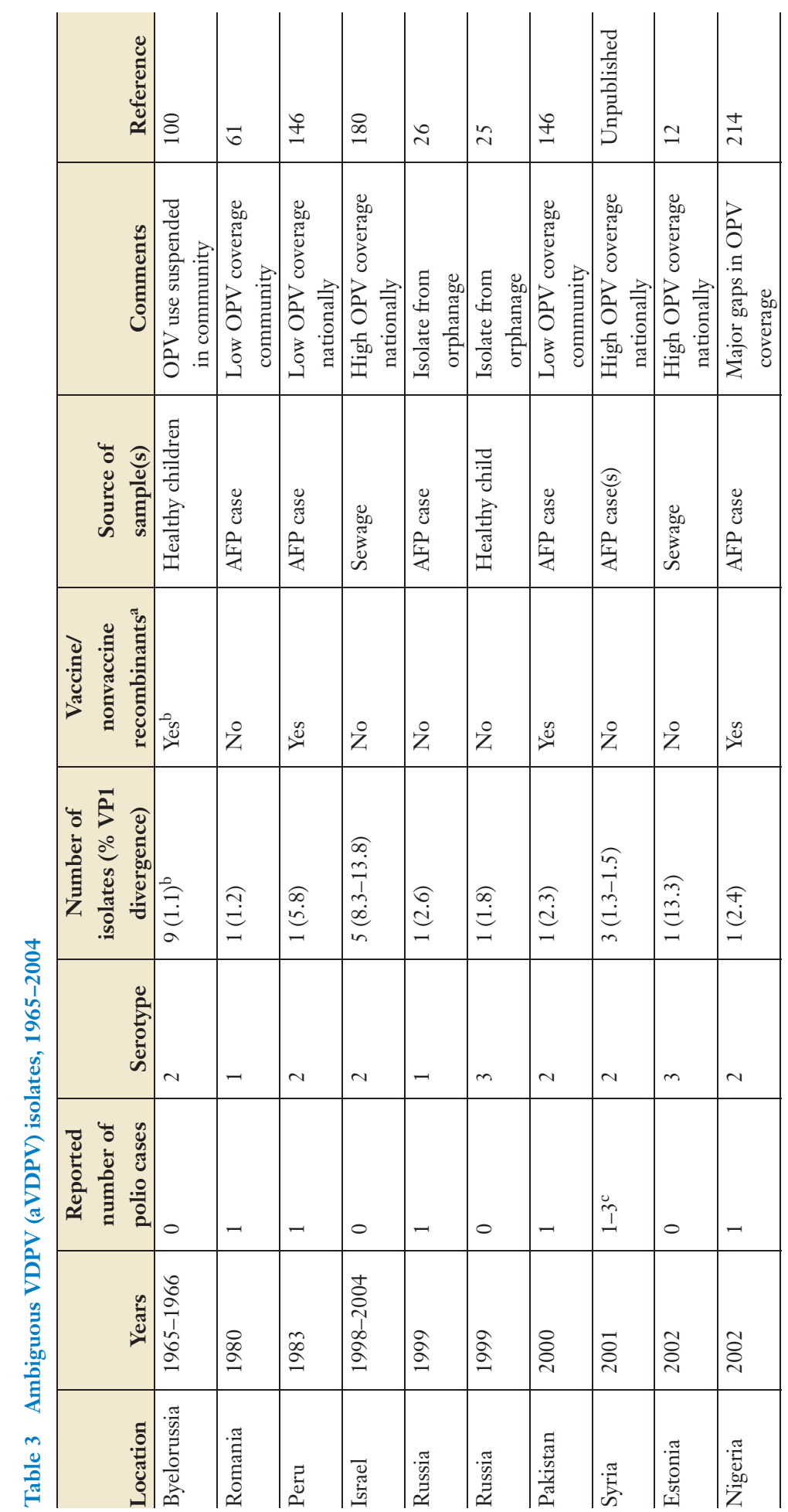




\begin{tabular}{|c|c|c|c|c|c|c|}
\hline 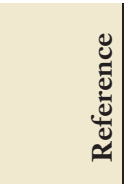 & $\stackrel{\sim}{ \pm}$ & $\stackrel{\partial}{\Xi}$ & $\stackrel{\bullet}{\vec{\sim}}$ & $\stackrel{ \pm}{\sim}$ & $\stackrel{0}{\sim}$ & 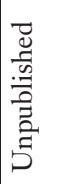 \\
\hline 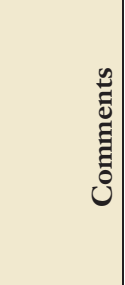 & 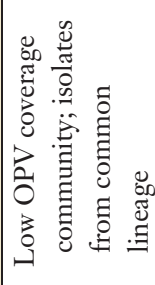 & 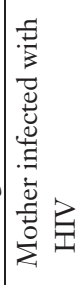 & 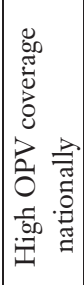 & 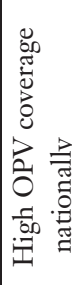 & 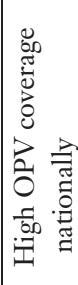 & 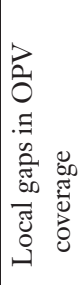 \\
\hline 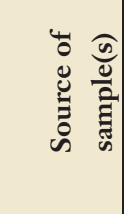 & 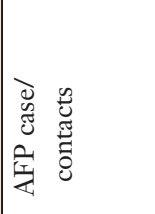 & 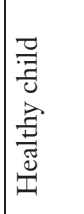 & 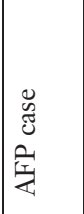 & 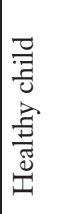 & 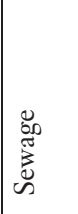 & 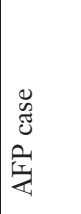 \\
\hline 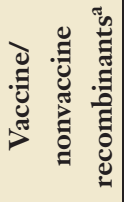 & $\stackrel{0}{Z}$ & $\stackrel{\circ}{Z}$ & $\stackrel{\circ}{Z}$ & $\stackrel{\circ}{Z}$ & $\stackrel{\circ}{Z}$ & $\stackrel{\circ}{Z}$ \\
\hline 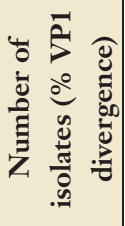 & $\underset{\infty}{\approx}$ & 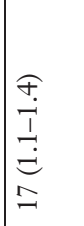 & 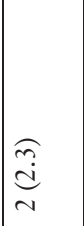 & 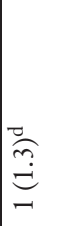 & 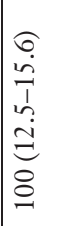 & $\underset{\mathrm{m}}{\stackrel{\overbrace{}}{\rightleftarrows}}$ \\
\hline 范 & - & - & $\sim$ & - & $\sim$ & $\sim$ \\
\hline 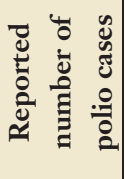 & - & 0 & - & 0 & 0 & - \\
\hline 怨 & $\underset{\sim}{\stackrel{\sim}{\sim}}$ & 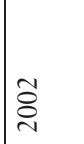 & 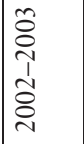 & $\stackrel{\text { }}{\stackrel{\sim}{े}}$ & 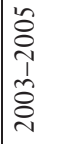 & $\underset{\sim}{\stackrel{+}{\Xi}}$ \\
\hline 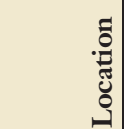 & 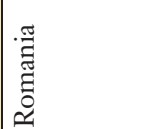 & 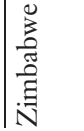 & 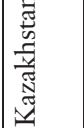 & 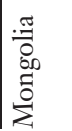 & 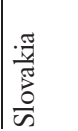 & 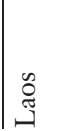 \\
\hline
\end{tabular}


(Table 3). The sequence relationships among the isolates were consistent with VDPV circulation for about a year. Stool surveys in neighboring low-coverage Roma communities detected no VDPVs, suggesting that VDPV circulation was highly localized (F. Delpeyroux, personal communication). It is unclear whether or how virus circulation could have been sustained for a year within such a small population.

Single VDPV isolates (all type 2) with genetic properties similar to those of the well-documented cVDPV outbreak isolates were obtained from AFP cases in Peru in 1983, Pakistan in 2000, and Nigeria in 2002 (Table 3) (Figure 6). The cases appeared at times of low OPV coverage in the affected communities, and in Pakistan and Nigeria several years after the eradication of the indigenous type 2 wild polioviruses. The isolates have been classified as aVDPVs (Table 3) because no other VDPVs were found in the communities. However, none of the patients were reported to be immunodeficient and all three isolates were vaccine/nonvaccine recombinants (see below).

\section{Risk Factors for cVDPV Emergence and Spread}

The most significant risk factor for cVDPV outbreaks, as for wild poliovirus outbreaks, is insufficient population immunity. The risk is also a function of other factors favoring poliovirus circulation: the number and density of nonimmune susceptible persons, the birth rate, deficiencies in hygiene/sanitation, and the seasonal duration of tropical conditions (135). The prior elimination of indigenous wild poliovirus circulation increases the risk because nonimmune susceptibles accumulate rapidly in the absence of high rates of poliovaccine coverage and naturally acquired immunity. The cVDPV outbreaks are similar to outbreaks from imported wild poliovirus, except that the cVDPV outbreak agents emerge endogenously. Virus excreted by OPV recipients may frequently recover the capacity for spread beyond immediate contacts, but spread is normally limited by population immunity. Outbreaks occur when the density of nonimmune susceptibles rises to the point at which the chains of cVDPV transmission can propagate $(55,135)$. The size of a cVDPV outbreak is a function of the size of the nonimmune population and the potential for transport of outbreak virus to susceptible communities elsewhere. Countries that were (or are) major reservoirs for wild poliovirus circulation, and where the potential for person-to-person poliovirus transmission is greatest, are at particularly high risk for cVDPV emergence, and maintenance of high rates of poliovaccine coverage in these settings is essential.

Each of the cVDPV outbreaks illustrates different aspects of the risk of cVDPV emergence. In Hispaniola, circulation appeared to be of longer duration and more widespread in Haiti, with its low rates of OPV coverage, than in the Dominican Republic, where cases were localized to pockets where OPV coverage rates were especially low. In Egypt, endemic VDPV circulation was prolonged and may have continued indefinitely had the rates of poliovaccine coverage not been raised. The finding of two distinct VDPV lineages in Madagascar demonstrates that VDPVs can emerge independently in localities where gaps in polio immunity arise. In the Philippines, VDPVs apparently circulated via a narrow chain of transmission localized to densely populated periurban slums with widening immunity gaps. By contrast, the localized VDPV circulation in a rural area of China with declining OPV coverage underscores the continuous risk of cVDPV outbreaks in populous countries where other risk factors exist. The cVDPV outbreak in Poland, and the detection of genetically divergent vaccine strains in Byelorussia, Romania, and Russia, demonstrate that vaccine virus progeny can also circulate in industrialized countries with temperate climates and moderate population densities if immunity gaps are sufficiently wide and other risk factors are present. 
An additional risk factor for cVDPV spread is insensitive surveillance. Apart from the outbreaks in the Philippines and Poland, and the localized, limited circulation in Guizhou, VDPV circulation had occurred in areas with low rates of AFP case reporting. VDPV circulation in Hispaniola, Madagascar, and the Philippines apparently began two to three years before the first cases were detected. VDPV circulation in Egypt occurred before implementation of AFP surveillance and was only detected retrospectively. The genetic diversity of the cVDPV isolates from Egypt and Haiti suggested that most poliomyelitis cases went unreported $(84,226)$, and the case counts probably greatly underestimate the disease burden of VDPV circulation in the two countries.

\section{Properties Shared by iVDPV and cVDPV Isolates}

In addition to the unusual degree of genetic divergence from their respective Sabin strains, VDPVs share other important properties. The most important of these is the capacity to cause paralytic polio in humans, documented for cVDPVs and for many, but not all, iVDPVs. Sequence analysis of both categories of VDPV isolates has shown that the critical determinants of the attenuated and temperature-sensitive phenotypes either have reverted or have been exchanged out by recombination. These genetic properties correlate with high neurovirulence for transgenic mice expressing the CD155 receptor $(12,84,113,117,119,169,177,180,223,226)$ and the capacity to replicate to high titers in cell culture at supraoptimal temperatures (12, 84, 117, 226). Moreover, all VDPV isolates characterized so far have antigenic properties distinct from the original Sabin strains (12, $71,84,117,177,180,226)$. The antigenic differences from the Sabin strains are less pronounced for type $2 \mathrm{cVDPVs}$ than for type 1 cVDPVs, possibly because selection against the Sabin 2 antigenic sites is less intense during replication in immunocompetent per- sons (133). While these experimentally determined properties may correlate with clinically relevant properties, they are not unique to VDPV isolates, as OPV-like polioviruses isolated from healthy individuals and VAPP patients may share some or all of these traits $(126,127,133)$.

\section{Properties Distinguishing iVDPV and cVDPV Isolates}

Despite their many similarities, iVDPVs and cVDPVs may differ in ways that reflect the different selective pressures exerted on the virus during person-to-person transmission compared with chronic infection in immunodeficient persons. These biological differences between isolates have permitted prediction of the identities of some aVDPV isolates, which were subsequently confirmed upon investigation of the associated cases (75; Y. Pongsuwanna, personal communication).

\section{Recombination with species $C$ en-} teroviruses (cVDPVs). All cVDPV isolates from the outbreaks in Egypt (226), Hispaniola (84), Madagascar (169), and the Philippines (177) were vaccine/nonvaccine recombinants, having capsid sequences derived from the parental vaccine strains and noncapsid sequences derived from nonvaccine viruses, most likely species C NPEVs, which are closely related to polioviruses (15). Several observations suggest that recombination may not be essential to the phenotypic reversion of OPV. First, the main determinants of attenuation of all three Sabin strains map to 5'-UTR and capsid region sites $(14,83$, $111,160,203)$ and most of the observed recombination sites map to the noncapsid region $(84,169,177,226)$. Second, serial recombination with species C NPEVs normally occurs during wild poliovirus circulation (66, 107), with the frequency of recombination being a function of the total number of mixed infections, which follows from the combined carriage rates of polioviruses and species $\mathrm{C}$ NPEVs. Evidence of serial recombination 
was found among the cVDPV isolates from Hispaniola (4 different recombinants detected) (84) and Egypt (22 different noncapsid region recombinants and 2 different $5^{\prime}$-UTR recombinants were detected) (226; C.-F. Yang, unpublished results). By contrast, the less divergent type $1 \mathrm{cVDPV}$ isolates from Guizhou had nonrecombinant genomes (W. $\mathrm{Xu}$, personal communication), and the 2002 aVDPV isolates from Romania were vaccine/vaccine recombinants sharing common recombination junctions (F. Delpeyroux, personal communication). Although both of these VDPVs had apparently spread within the community via person-to-person transmission, the duration of circulation and the extent of spread may have been too limited to favor the emergence of vaccine/nonvaccine recombinants.

None of the iVDPV isolates described thus far have been vaccine/nonvaccine recombinants, although several have been vaccine/vaccine recombinants. This observation applies even to iVDPV isolates with highly divergent genomes that probably have been replicating for many years $(60,88,113,117$, 223). Vaccine/nonvaccine recombinants are rare among OPV-like isolates $(33,91)$, presumably because of limited opportunities for mixed infection with species $\mathrm{C}$ enteroviruses. Therefore, given these correlations and uncertainties, if a VDPV has evidence of recombination with a group C NPEV, it likely is a cVDPV and the associated case should be urgently investigated.

Extensive antigenic variability (iVDPVs). Antigenic evolution normally occurs during poliovirus replication $(31,129)$. However, evolution appears to occur more slowly among circulating wild polioviruses than among vaccine-related strains. Antigenic evolution is especially pronounced for the Sabin 1 and Sabin 3 strains during replication in healthy OPV recipients $(31,129,133)$ and less pronounced for the Sabin 2 strain (133). Although the cVDPV isolates from Egypt, Hispaniola, and the Philippines were anti- genic variants of the Sabin strains, variability among the surface determinants was limited despite the high degree of nucleotide divergence among many of the isolates (177; J. Shaw, C. Burns, J. Jorba \& C.-F. Yang, unpublished results). By contrast, the neutralizing antigenic sites of iVDPV isolates are frequently highly divergent and variable, and immunodeficient prolonged excretors may be infected with more than one antigenic variant $(88,117,146,223)$. Indeed, the antigenic sites of some type $2 \mathrm{iVDPV}$ isolates are more divergent from Sabin 2 than are those of the unrelated isolate, MEF-1 (146). Therefore, VDPV isolates, especially of serotypes 2 and 3, that show extensive divergence at neutralizing antigenic sites are candidate iVDPVs.

Multiple, coreplicating lineages (iVDPVs). The virus populations in isolates of circulating wild polioviruses and cVDPVs typically have low genetic diversity. By contrast, iVDPV isolates from individual patients are frequently mixed populations of variants, all derived from a single initiating OPV dose. Some iVDPV populations can be quite complex. For example, one type 1 iVDPV had two main lineages (88), another type $1 \mathrm{iVDPV}$ had five observed lineages (223), a type $2 \mathrm{iVDPV}$ from the United States had two main lineages (146), a type 3 iVDPV from the United Kingdom also contained diverging lineages (117), and type 2 isolates from an immunodeficient patient and contacts had heterogeneous capsid sequences (27). Clinical isolates containing mixed VDPV populations are likely iVDPVs, whereas environmental isolates containing mixed VDPV populations remain more difficult to classify, as they may indicate either excretion by a single immunodeficient person or person-to-person spread.

Person-to-person transmission (cVDPVs). The cVDPVs have spread by person-to-person transmission, whereas this has not been shown for iVDPVs. This does not imply that iVDPVs would not, under 
suitable conditions, recover the capacity for efficient person-to-person transmission. However, most reported iVDPV cases have been from countries with high rates of poliovaccine coverage and high levels of sanitation and hygiene, conditions unfavorable for extensive poliovirus transmission (Table 1).

\section{aVDPVs Reconsidered}

The differing properties of iVDPV and cVDPV isolates provide insights into the likely identities of some aVDPV isolates (Table 3). For example, the highly evolved environmental VDPV isolates from Israel (180; L. Shulman, personal communication), Estonia (12), and Slovakia (T. Hovi \& E. Gavrilin, personal communication) are either nonrecombinant or vaccine/vaccine recombinants. Moreover, all have highly modified neutralizing antigenic sites. Although the Israel and Slovakia isolates each comprise several distinct lineages derived from a common ancestor, this may be an indication of the excellent sensitivity of environmental surveillance for wild polioviruses and VDPVs (48, $115)$, rather than of wider VDPV circulation. The environmental aVDPV isolates are much more likely to be iVDPVs than cVDPVs, as the rates of OPV coverage in all three countries are high. The Slovakia VDPVs may soon be unambiguously identified, as the source of the virus has been systematically traced to a specific block of apartments, increasing the opportunities to identify and offer medical assistance to the suspected chronic excretor (T. Hovi \& E. Gavrilin, personal communication).

As suggested above, the type 2 aVDPVs from Peru (1983), Pakistan (2000), and Nigeria (2002) are probably cVDPVs that had been in circulation for two years or more (Table 3). All are vaccine/nonvaccine recombinants, and all were isolated from communities where the risk factors for poliovirus circulation converge and where persons with primary immunodeficiencies would be ex- pected to have short survival times. Similarly, the aVDPV isolates from Byelorussia (1965-1966) and Romania (1980 and 2002) may have signaled localized community circulation (Table 3). The finding that one of the type 2 isolates from Byelorussia was a vaccine/nonvaccine recombinant is consistent with circulation (100). The two 1999 aVDPVs from Russia were isolated from different children living in the same orphanage, and although the isolates were of different serotypes, they shared some vaccine/vaccine recombinant noncapsid sequences $(25,26)$. One possibility is that the crowded and poor sanitary conditions of the orphanage facilitated person-to-person spread of VDPVs, which may have originated from a single chronically infected child.

The distinction between some aVDPVs and the recognized cVDPVs may depend as much on community risk factors as on the precise biological properties of the VDPVs. Some aVDPVs might have spread more widely (and thus be recognized as cVDPVs) had the susceptible populations been larger and had there been more frequent contact between infected and susceptible populations. Some aVDPVs might also have been recognized to be cVDPVs if surveillance had been more sensitive. The properties of some aVDPV isolates, and even some OPV-like isolates in certain settings $(27,100)$, may yield insights into the early dynamics of cVDPV emergence which in other settings could result in an outbreak.

\section{Independence of iVDPVs and cVDPVs}

The shared properties of iVDPV and cVDPV isolates have prompted the suggestion that the recognized cVDPV outbreaks may have been triggered by iVDPV chronic excretors. Because the early events in cVDPV outbreaks have not been observed (except possibly in Guizhou and Romania, 2002), it is difficult to rule out this possibility rigorously. However, two key observations suggest that iVDPV 
infections and cVDPV outbreaks have so far been independent. First, cVDPV outbreaks have occurred in communities where the expected survival times for persons with B-cell immunodeficiencies are short (69). Second, the extent of amino acid substitution in the neutralizing antigenic sites is typically lower for $\mathrm{cVDPV}$ isolates than for iVDPV isolates having similar levels of total capsid sequence divergence from the vaccine (J. Jorba, unpublished results). Past experience, however, may not be a predictor of future conditions, as iVDPVs from chronically infected individuals may present a risk for spread into the community in some settings in the post-OPV era, and additional surveillance measures may then be needed to detect long-term iVDPV excretors (see below).

\section{Laboratory Surveillance for VDPVs}

Laboratory-based surveillance for VDPVs began in 1997 in the Americas, with the sequencing of the VP1 genes of poliovirus isolates from AFP cases throughout the region (34). Unfortunately, this approach did not provide an early warning of the Hispaniola cVDPV outbreak because no polioviruses were isolated there in the preceding five years (84). Following that outbreak, intensive screening of recent poliovirus isolates for cVDPVs was initiated by laboratories within the WHO Global Polio Laboratory Network (209, 214). Beginning in 2001, all poliovirus isolates from AFP cases have been screened by two different standard ITD methods [one molecular, usually diagnostic PCR (224), and one antigenic, usually the ELISA (198)] for evidence of prolonged replication or circulation $(209,214)$. Any poliovirus isolate without concordant vaccinelike results in the two ITD tests and all isolates showing "nonvaccine-like" antigenic properties are further characterized by VP1 sequencing $(209,214,216)$. WHO is notified of any current isolates having $\geq 1 \% \mathrm{VP} 1$ divergence, and both the case and the associated isolate are promptly investigated further while prepara- tions are made for any necessary response immunization activities.

Between 1999 and June 2004, vaccinerelated isolates from over 10,000 AFP cases from all WHO regions were screened for VDPVs $(209,214,216)$. The large majority (>95\%) of isolates had vaccine-like antigenic properties and were usually not investigated further. Of the remainder, 42 were cVDPVs (all from the four recent outbreaks), 6 were iVDPVs, and 12 were aVDPVs (209, 214, 216). The additional aVDPVs were found in healthy children and in sewage $(214,216)$ (Table 3).

The underlying rationale for the screening method is that vaccine-related isolates obtained from specimens taken within a few weeks of the initiating OPV dose are less likely to be enriched for antigenic variants than are isolates from later specimens. However, the rates and extent of antigenic evolution as measured by the ELISA differ among the three Sabin strains in the approximate order, type $1>$ type $3>$ type 2 . Most type 1 and type 3 antigenic variants are genetically OPV-like, with VP1 sequence drift $<1 \%$. By contrast, the type $2 \mathrm{cVDPV}$ from Madagascar showed only limited antigenic divergence.

Two new molecular approaches that screen for VDPVs have recently been developed. The first approach, based upon microarrays sampling comprehensively across the genome, can detect genetic divergence or recombination in vaccine-related isolates by oligonucleotide duplex stability (25). This technique can detect genetic divergence below $1 \%$ and has been used to identify VDPVs (25) and less drifted recombinant OPVlike isolates $(27,100)$. The second approach, based upon sampling $2 \mathrm{C}$ and $3 \mathrm{D}$ sequences by PCR, can detect most vaccine/vaccine and vaccine/nonvaccine recombinants using routine procedures of the WHO Global Polio Laboratory Network (91).

Investigations into the cVDPV outbreaks in Hispaniola, Madagascar, and Guizhou were initially prompted by the unusual epidemiologic finding of temporal and geographic 
clustering of AFP cases associated with vaccine-related isolates of a single serotype. Occurrences of type-specific clustering of vaccine-related isolates require urgent investigation of both cases and isolates.

\section{The Surprising Serotype Distribution for VDPVs}

The current listing of VDPV events (Tables 1-3) shows a serotype distribution (33\% type $1,60 \%$ type 2 , and $7 \%$ type 3 ) different from that for VAPP cases, which are predominantly associated with type 3 and rarely associated with type 1 (51, 183). The serotype distribution of cVDPV events is similar to that for iVAPP and prolonged iVDPV infections. The increased risk for type 2 VDPVs probably results from the greater tendency of the type 2 vaccine strain to spread to household (11, $55)$ and community contacts $(8,11,55$, 185). The increased risk for type 1 VDPVs may occur because prolonged replication increases the likelihood of full reversion of the multiple determinants of the attenuated and temperature-sensitive phenotypes (64), and the high attack rates (135) and efficient dissemination of type 1 poliovirus infections (147) may increase chances for detecting type 1 VDPVs. However, the rarity of type 3 VDPVs was unexpected, in view of the particular genetic instability of the Sabin 3 strain and its predominant contribution to VAPP. The reduced association of Sabin 3 with contact VAPP compared with recipient VAPP probably reflects the lower transmissibility of the Sabin 3 strain. Moreover, the only documented case associated with a type 3 iVDPV was in a child fed monovalent type 3 OPV (112), and the one iVAPP case in the United States infected with Sabin 3 was also concurrently infected with Sabin 1 and Sabin 2 (186). One possible explanation for these observations is that Sabin 3 is overgrown by more rapidly replicating derivatives of Sabin 1 and Sabin 2 in the intestines of immunodeficient hosts, but such overgrowth is normally suppressed by a robust immune response in immunocompetent hosts.

\section{VDPVs AND THE ENDGAME STRATEGY FOR POLIO ERADICATION}

\section{Pre- and Posteradication Challenges}

The restriction of indigenous wild poliovirus circulation to only a few parts of the world has greatly reduced the risk of poliovirus importation. However, this favorable circumstance places increased importance on polio immunization to maintain population immunity in countries that are now polio-free. In areas where the risks for cVDPV emergence are highest, periodic NIDs or SNIDs may be necessary to close any gaps in population immunity that may arise from deficiencies in routine OPV coverage. The required frequency and extent of the immunization campaigns is determined by the magnitude of the risk factors for poliovirus circulation in each population. In the current pre-eradication phase, the same strategies implemented to limit the spread of imported wild polioviruses would similarly limit the spread of VDPVs, and all poliovirus outbreaks can be controlled by mass immunization campaigns using OPV. The greatest challenges arise in the posteradication phase, when the risks of wild poliovirus infection are greatly reduced, public concern about polio as a disease wanes, population immunity can be maintained only by immunization, and the only continuous source of poliovirus infection and polio adverse events is from OPV. While the original plan for global polio eradication envisioned a straightforward endgame strategy (80), that phase has become far more complex in view of the risks presented by VDPVs.

\section{Eradication Principles Revisited}

The occurrence of VDPVs appears to violate two key biological assumptions for 
poliovirus eradication: (a) the absence of a persistent carrier state and $(b)$ the implicit assumption that the vaccine would not circulate and spread (55). When these principles were first explicitly detailed in 1997 (42), few examples of immunodeficient long-term chronic excretors had been described, and in every known case excretion of vaccinederived poliovirus had stopped. Moreover, the cVDPV outbreaks in Hispaniola, the Philippines, and Madagascar had not yet occurred, and the cVDPV outbreak in Egypt had not yet been recognized. The subsequent recognition of long-term iVDPV excretors and the potential for cVDPV emergence has prompted in depth reassessment of the endgame strategies for the Global PEI $(192,221)$.

\section{Limited Applicability of the Smallpox Model}

The only infectious disease to have been successfully eradicated so far is smallpox and its agent, variola virus (52). Although the Smallpox Eradication Program inspired and shaped the Global PEI, the clinical and epidemiologic differences between the two diseases and the marked biological differences between variola virus and poliovirus have limited the applicability of the smallpox model to the polio endgame strategy (73). For example, all variola infections cause clinically apparent symptoms, whereas the large majority of wild poliovirus infections are inapparent. Moreover, the clinical signs of smallpox are unique, whereas there are many etiologies for AFP. Variola virus exists as a single serotype, in contrast to the three poliovirus serotypes. The smallpox vaccine is vaccinia, a genetically stable, avirulent DNA virus antigenically related to, but distinct from, variola. Personto-person spread of vaccinia virus is rare, because the vaccine is inoculated subcutaneously and there is no respiratory transmission as with smallpox. By contrast, the use of mutable live polioviruses in OPV administered by the natural route of infection present spe- cial challenges not encountered with smallpox eradication (43). The endgame strategy for smallpox eradication was straightforward: Immunization against smallpox could stop before global smallpox eradication in countries where the risks of smallpox importation had become negligible. After smallpox eradication, widespread immunization could stop, but targeted immunization of highrisk groups (for example, laboratory workers) could continue. There was no need for a detailed global endgame strategy for the Smallpox Eradication Program.

\section{Early Alternative Endgame Scenarios}

As the Global PEI has progressed, planning for the posteradication phase intensified (16), the risks for polio re-emergence were critically assessed (55), and various posteradication scenarios were considered $(43,206)$. Six different scenarios were initially described: (a) indefinite use of OPV, (b) transition to use of IPV, (c) coordinated cessation of OPV use, (d) uncoordinated (country-by-country) cessation of OPV use, (e) sequential removal of Sabin serotypes from OPV, and $(f)$ development of new OPV strains (206). These scenarios have been reassessed in view of recent experience and current understanding of the risks presented by VDPVs $(46,56)$. Coordinated cessation of OPV use has emerged as the preferred scenario. The key advantages and disadvantages of each scenario are briefly described below.

Indefinite use of OPV. The potential advantage of indefinite OPV use in countries currently using OPV would be the maintenance of population immunity, thereby affording protection from reintroduced wild poliovirus or the spread of iVDPVs. The disadvantages would be a sustained annual incidence of up to 500 cases of VAPP, and the necessity to maintain indefinite public investment in and community support for immunization against a disease that had been certified as eradicated. The practical challenge 
to sustain adequate levels of OPV coverage even in the current era was underscored by the recent cVDPV outbreaks. Indefinite use of OPV is not a viable policy because it is likely that OPV coverage rates would fall below safe levels in many countries because of competing health priorities (as in Madagascar), because of social instability (as in Haiti), or because susceptibles may accumulate in the highest risk communities (as in the Philippines).

Transition to use of IPV. Transition to exclusive use of IPV eliminates the risk of new cases of VAPP (3) and new iVDPV infections and has the potential to maintain population immunity in many countries. Global transition to IPV carries several potential disadvantages, including (a) unknown efficacy in stopping poliovirus transmission even at high rates of coverage in tropical developing country settings (because of inefficient and transient induction of intestinal immunity) (187), (b) difficulties in integrating the optimal IPV routine immunization schedule into the WHO schedule for other vaccines, $(c)$ severe deficiencies in routine immunization coverage in many developing countries, and (d) the potential to reduce the sensitivity of AFP surveillance (because the early stages of poliovirus circulation might be obscured by an increased proportion of inapparent infections). In addition, there is the ongoing risk of exposure of workers in IPV production facilities to virulent wild poliovirus seed strains. IPV production and use will likely continue in many high-income countries where vaccine can be produced under the appropriate levels of biological containment (44, 212). However, IPV production without the necessary containment facilities or production in countries unlikely to maintain high levels of poliovaccine coverage present unacceptable risks. These risks are not eliminated by the use of Sabin strains for IPV production (40) because of the potential for reversion of the attenuated strains upon accidental infection of a worker.
Country-by-country cessation of OPV use. The primary appeal of this scenario is that it adheres to the principle that each country should develop its own immunization policies. However, as illustrated by the Hispaniola outbreak, this scenario presents unacceptable risks for emergence of cVDPVs, as persons infected with OPV virus could reseed countries (or communities) where polio immunization had stopped.

Coordinated cessation of OPV use. A major advantage of this scenario is that OPV use would cease at a time when population immunity to polio would be highest, thereby minimizing the risks for cVDPV emergence. Potential disadvantages of this and any other scenario envisioning cessation of OPV use (which may result in cessation of all polio immunization in many developing countries) is the rapid accumulation of a nonimmune cohort susceptible to polio, the potential loss of global capacity to produce OPV, and the need for large stockpiles of OPV if any polio cases or outbreaks appeared after OPV use had stopped (see below).

\section{Sequential removal of Sabin strains from} OPV. A variation on coordinated cessation would be the sequential removal of Sabin strains from OPV as the corresponding wild poliovirus serotypes are eradicated (29). In this scenario, coordinated transition would first be to a bivalent (type $1+$ type 3 ) OPV, followed by coordinated transition to a monovalent $\mathrm{OPV}$, and then to the sole remaining serotype (most likely type 1 ). Coordinated transition to a bivalent OPV would permit a critical type-specific test of the coordinated OPV cessation strategy, especially because derivatives of the Sabin 2 strain have the highest potential for secondary spread and represent the VDPV serotype most frequently found. A variation on this approach would be to use monovalent vaccines to the surviving serotype(s). Despite its scientific appeal (55), this approach faces three key obstacles: (a) securing regulatory approval for a type $1+$ 
3 bivalent OPV may prove difficult, as it would represent an untested OPV product with no record of performance in the field; (b) logistical implementation of simultaneous withdrawal of all preceding OPV formulations will likely be arduous; and $(c)$ the urgency to stop OPV use (see below) may not favor implementation of a more protracted endgame strategy.

Development of new OPV strains. It would be highly desirable to apply the dramatic advances in the understanding of the molecular mechanisms for attenuation of the Sabin strains to the development of safer, more effective, and genetically more stable OPV strains. Use of the engineered Sabin strain variants already developed (1, 62, 97, $110,137)$ might greatly reduce the incidence of VAPP. Less clear is to what extent these new strains could reduce the risk of emergence of VDPVs. VDPVs with increased replicative fitness might still arise (but possibly at much lower frequencies) through loss of the engineered IRES sequences by recombination, especially in countries with high rates of enterovirus carriage. Moreover, it is difficult to predict the evolution of any engineered constructs during chronic infection of an immunodeficient person. Nonetheless, new OPV strains would likely offer multiple advantages over the current Sabin strains. The critical challenge to the introduction of new attenuated OPV strains would be the design and implementation of field trials sufficiently large to detect a reduction from the current VAPP risk or from the even less readily quantifiable VDPV risk.

\section{Current Endgame Perspectives and Challenges}

After review of the threat that VDPVs present to polio eradication $(7,46,56,211)$, WHO has outlined a strategic plan for cessation of all routine use of $\mathrm{OPV}(215,220)$. The strategy has the following components: (a) Routine OPV use will stop in a globally syn- chronized manner approximately two to three years after the last detection of wild poliovirus, (b) IPV will not be used routinely in all countries, (c) AFP and poliovirus surveillance will be maintained for at least three years after detection of the last cVDPVs, (d) all laboratory stocks of poliovirus will be contained upon discontinuation of OPV, and (e) a global OPV stockpile and response capacity will be established to stop any poliovirus transmission that may occur after cessation of OPV use. Despite the urgent need to stop wild poliovirus circulation as quickly as possible, the WHO endgame strategy is developing rapidly.

Interruption of wild poliovirus transmission. This is the highest priority for the Global PEI (192), and immunization and surveillance activities have been intensified in the remaining reservoir areas. The prior eradication of indigenous wild poliovirus type 3 in Egypt has opened the way for use in that country of monovalent type $1 \mathrm{OPV}$, which is expected to increase the efficacy of each OPV dose (167). This strategy has also been applied in India in 2005, and may be applied in Pakistan and Afghanistan, and in West Africa upon eradication of wild poliovirus type 3 .

Certification of wild poliovirus eradication. WHO has established a formal process for the certification of wild poliovirus eradication (181). Independent National Certification Committees report to Regional Certification Commissions, who in turn report to the Global Commission for the Certification of Poliomyelitis Eradication. The main requirement for certification is the absence of any isolation of wild poliovirus from patients with AFP, healthy individuals, or the environment for at least three years in the presence of highquality surveillance. Substantial progress toward containment of wild poliovirus laboratory stocks is also required (see below). The findings of the Regional Commissions of the three Regions declared polio-free (Americas, 
1994; Western Pacific, 2000; Europe, 2002) have been reaffirmed by the absence of detection of indigenous wild poliovirus in the following years. Surveillance must be dramatically strengthened in some endemic countries to meet the strict requirements for certification.

Synchronous cessation of OPV use. Ideally, OPV use could cease after a series of Global Immunization Days in countries using OPV. Short of that, cessation should be coordinated among countries to be completed within a few weeks, and reliable mechanisms established in all countries to assure that all OPV stocks in the field are recalled and destroyed. It is critical that all countries agree that OPV will never again be used except in the event of an outbreak. WHO is preparing guidelines to help national governments develop plans for OPV cessation (220).

Containment of poliovirus laboratory stocks. Containment of poliovirus materials in the laboratory is an essential safeguard to the achievement of global polio eradication. WHO has developed a comprehensive Global Action Plan for poliovirus containment in the pre-eradication, posteradication, and post-OPV eras (212). In the current pre-eradication phase, biomedical laboratories worldwide are being surveyed to identify those storing wild poliovirus infectious or potential infectious materials. $\mathrm{Na}$ tional inventories of laboratories retaining such materials are forwarded to the Certification Commissions of each WHO Region. Laboratories are instructed to institute enhanced BSL-2/polio measures for safe handling of wild poliovirus. One year after isolation of the last wild poliovirus, biomedical laboratories will be notified of the likelihood of global polio eradication, and those on national inventories will be required to choose among three options: (a) implement BSL-2/polio or BSL-3/polio biosafety measures as appropriate, $(b)$ transfer their stocks to laboratories implementing the higher biosafety measures, or $(c)$ destroy their wild poliovirus stocks. In the post-OPV era, individuals exposed to any poliovirus, including OPV strains, could potentially transmit the virus to nonimmune susceptible persons in the community (212).

Compliance with the Global Action Plan has been excellent. In the United States, the country with the largest number of individual biomedical laboratories, all 5585 institutions and laboratories judged most likely to possess and may possess wild-polioviruscontaining materials responded to the survey, and of these, 180 reported retention of wild poliovirus stocks or material potentially containing wild poliovirus (24). All countries in the European Region have completed the survey and inventory phase as part of their certification process, as have most countries in the Western Pacific Region. Laboratory containment activities are underway in all other WHO Regions (44).

When wild poliovirus transmission is interrupted and OPV use stops, the need for containment of wild and OPV (Sabin) polioviruses will converge. The consequences of laboratory-associated transmission are too great to depend solely on voluntary compliance. Strong international biosafety measures and global placement of limits on the number of laboratories retaining poliovirus materials are crucial to minimize the risk of transmitting poliovirus from the laboratory to growing susceptible communities.

Establishment of a global OPV stockpile. It is necessary for WHO, in coordination with some national governments, to establish a global stockpile of monovalent OPV to permit a type-specific response to any future poliovirus circulation (220). Regional stockpiles will be strategically located to permit rapid response to any post-OPV polio outbreak. The anticipated global stockpile will be of sufficient size to immunize several annual global birth cohorts, permitting response to outbreaks in populous countries several years
BSL: biosafety level 
after OPV cessation. Because OPV production will likely stop after OPV cessation, the stockpile must be produced before that time to assure adequate supplies and to avoid production of OPV under enhanced biosafety level conditions. If future conditions warrant reestablishment of OPV production, the stockpile must be large enough to protect the world's population until the newly produced OPV becomes available.

Detection and control of iVDPV infections. The number of long-term iVDPV excretors is expected to steadily decline in countries that have shifted to IPV. The continued presence of iVDPV excretors may not represent a serious health risk within countries that maintain high rates of IPV coverage. By contrast, iVDPV excretors in middle-income countries or within high-income enclaves in low-income countries could present a serious health risk in the post-OPV era. It is important that these risks be properly assessed (46) and that surveillance strategies are developed to detect iVDPV infections wherever they may occur (see below). Effective treatments for clearing iVDPV infections must also be found. Although attempts to clear one chronic iVDPV infection by combined treatment with oral immunoglobulin and pleconaril (a firstgeneration antiviral effective against some poliovirus strains) were unsuccessful (113), alternative protocols and the use of newer antiviral agents may yield more promising results.

Maintenance of global poliovirus surveillance. Because poliovirus could be reintroduced into the population by the emergence and spread of VDPVs, by a breach of containment in a laboratory or poliovaccine production facility, or even by intentional release, it is essential that poliovirus surveillance is maintained for the foreseeable future. Immediately after cessation of OPV use, it will be necessary to intensify poliovirus surveillance, especially in the traditional reservoirs for wild poliovirus circulation where the biological risks for poliovirus circulation (and potentially of cVDPV emergence) are highest. This would include populous, tropical, developing countries whose effective immunization programs had overcome the inherent biological risks. The sensitivity of environmental surveillance in developing countries would increase because the high background of OPV-like viruses would fade. The technical aspects of poliovirus surveillance would simplify six months into the post-OPV period, as any poliovirus isolate would raise concerns and be regarded as a potential public health emergency, especially if there is any geographic clustering of isolates of the same serotype. If, as is hoped, the early post-OPV years prove uneventful, it will still be necessary to maintain surveillance for AFP cases and poliovirus infections in the coming years. AFP and poliovirus surveillance will be integrated into other disease surveillance activities (213) as efforts to control other vaccine-preventable diseases broaden and as the global network of laboratories first built to support polio eradication extends its support to wider infectious disease control initiatives.

\section{CONCLUSIONS}

The era of wild polioviruses is rapidly drawing to a close. In a short time it appears likely that the only source of poliovirus infection worldwide will be from OPV. Successful navigation from the current pre-eradication era to the imminent post-OPV era and beyond requires surmounting an unprecedented series of public health challenges. The first step is the elimination of the last reservoirs of wild poliovirus circulation. Soon thereafter, implementation of the posteradication endgame strategy can begin. Implementation of this crucial phase of polio eradication requires a more detailed assessment of the risks of VDPV emergence in various settings (especially those at highest risk); a clearer understanding of the biological properties of VDPVs; reinforcement of global poliovirus surveillance; development of effective means to clear long-term iVDPV 
infections; establishment of appropriately formulated, sized, and positioned OPV stockpiles; and completion of poliovirus contain- ment worldwide. Moving forward will continue to require the best efforts of the global public health and scientific communities.

\section{ACKNOWLEDGMENTS}

We thank Edson da Silva, Francis Delpeyroux, Gene Gavrilin, Tapani Hovi, Javier Martín, Philip Minor, Yaowapa Pongsuwanna, Hiroyuki Shimizu, Lester Shulman, Hamideh Tabatabei, Wenbo $\mathrm{Xu}$, and David Wood for sharing their results prior to publication, Paul Chenoweth for preparing high-resolution maps of current polio cases, and the virologists of the WHO Global Polio Laboratory Network for sharing specimens and information with us.

\section{LITERATURE CITED}

1. Agol VI, Pilipenko EV, Slobodskaya OR. 1996. Modification of translational control elements as a new approach to design of attenuated picornavirus strains. F. Biotechnol. 44:119-28

2. Alexander JP Jr, Gary HE Jr, Pallansch MA. 1997. Duration of poliovirus excretion and its implications for acute flaccid paralysis surveillance: a review of the literature. 7 . Infect. Dis. 175(Suppl. 1):S176-82

3. Alexander LN, Seward JF, Santibanez TA, Pallansch MA, Kew OM, et al. 2004. Vaccine policy changes and epidemiology of poliomyelitis in the United States. FAMA 292:1696701

4. Andrus JK, de Quadros C, Olivé JM, Hull HF. 1992. Screening of cases of acute flaccid paralysis for poliomyelitis eradication: ways to improve specificity. Bull. WHO 70:59196

5. Andrus JK, Strebel PM, de Quadros CA, Olivé JM. 1995. Risk of vaccine-associated paralytic poliomyelitis in Latin America, 1989-91. Bull. WHO 73:33-40

6. Anonymous. 1980. Studies on new attenuated strains of type III live poliomyelitis vaccine. I. Development of a new type III attenuated poliovirus. Chin. Med. F. 93:58390

7. Aylward RB, Cochi SL. 2004. Framework for evaluating the risks of paralytic poliomyelitis after global interruption of wild poliovirus transmission. Bull. WHO 82:40-46

8. Aylward RB, Porta D, Fiore L, Ridolfi B, Chierchini P, Forastiere F. 1997. Unimmunized Gypsy populations and implications for the eradication of poliomyelitis in Europe. 7 . Infect. Dis. 175(Suppl. 1):S86-88

9. Balanant J, Guillot S, Candréa A, Delpeyroux F, Crainic R. 1991. The natural genomic variability of poliovirus analyzed by a restriction fragment polymorphism assay. Virology 184:645-54

10. Bellmunt A, May G, Zell R, Pring-Akerblom P, Verhagen W, Heim A. 1999. Evolution of poliovirus type I during 5.5 years of prolonged enteral replication in an immunodeficient patient. Virology 265:178-84

11. Benyesh-Melnick M, Melnick JL, Rawls WE, Wimberley I, Barrera-Oro J, et al. 1967. Studies on the immunogenicity, communicability, and genetic stability of oral poliovaccine administered during the winter. Am. F. Epidemiol. 86:112-36

12. Blomqvist S, Savolainen C, Laine P, Hirttio P, Lamminsalo E, et al. 2004. Characterization of a highly evolved vaccine-derived poliovirus type 3 isolated from sewage in Estonia. 7. Virol. 78:4876-83 
13. Bodian D, Morgan IM, Howe HA. 1949. Differentiation of types of poliomyelitis viruses. III. The grouping of fourteen strains into three basic immunologic types. Am. F. Hyg. 49:234-45

14. Bouchard MJ, Lam DH, Racaniello VR. 1995. Determinants of attenuation and temperature sensitivity in the type 1 poliovirus Sabin strain. 7. Virol. 69:4972-78

15. Brown BA, Oberste MS, Maher K, Pallansch M. 2003. Complete genomic sequencing shows that polioviruses and members of human enterovirus species $\mathrm{C}$ are closely related in the non-capsid coding region. 7. Virol. 77:8973-84

16. Brown F. 2001. Progress in polio eradication: vaccine strategies for the end game. Dev. Biol::105

17. Cabasso VJ, Jervis GA, Moyer AW, Roca-Garcia M, Orsi EV, Cox HR. 1959. Cumulative testing experience with consecutive lots of oral poliomyelitis vaccine. In Live Poliovirus Vaccines. 1st Int. Conf. Live Poliovirus Vaccines, pp. 102-34. Washington, DC: Pan Am. Sanit. Bur.

18. Cammack N, Phillips A, Dunn G, Patel V, Minor PD. 1988. Intertypic genomic rearrangements of poliovirus strains in vaccinees. Virology 167:507-14

19. Cent. Dis. Control Prevent. 1994. Certification of poliomyelitis eradication-the Americas, 1994. Morbid. Mortal. Wkly. Rep. 43:720-22

20. Cent. Dis. Control Prevent. 1997. Poliomyelitis prevention in the United States: introduction of a sequential vaccination schedule of inactivated poliovirus vaccine followed by oral poliovirus vaccine. Recommendations of the Advisory Committee on Immunization Practices (ACIP). Morbid. Mortal. Wkly. Rep. 46(No. RR-3):1-25

21. Cent. Dis. Control Prevent. 2000. Developing and expanding contributions of the Global Laboratory Network for Poliomyelitis Eradication, 1997-1999. Morbid. Mortal. Wkly. Rep. 49:156-60

22. Cent. Dis. Control Prevent. 2001. Certification of poliomyelitis eradication-Western Pacific Region, October 2000. Morbid. Mortal. Wkly. Rep. 50:1-3

23. Cent. Dis. Control Prevent. 2002. Certification of poliomyelitis eradication-European Region, June 2002. Morbid. Mortal. Wkly. Rep. 51:572-74

24. Cent. Dis. Control Prevent. 2004. National laboratory inventory for global poliovirus containment-United States, November 2003. Morbid. Mortal. Wkly. Rep. 53:457-59

25. Cherkasova E, Laassri M, Chizhikov V, Korotkova E, Dragunsky E, et al. 2003. Microarray analysis of evolution of RNA viruses: evidence of circulation of virulent highly divergent vaccine-derived polioviruses. Proc. Natl. Acad. Sci. USA 100:9398-403

26. Cherkasova EA, Korotkova EA, Yakovenko ML, Ivanova OE, Eremeeva TP, et al. 2002. Long-term circulation of vaccine-derived poliovirus that causes paralytic disease. F. Virol. 76:6791-99

27. Cherkasova EA, Yakovenko ML, Rezapkin GV, Korotkova EA, Ivanova OE, et al. 2005. Spread of vaccine-derived poliovirus from a paralytic case in an immunodeficient child: an insight into the natural evolution of oral polio vaccine. F. Virol. 79:1062-70

28. Chumakov KM, Norwood LP, Parker ML, Dragunsky EM, Ran YX, Levenbook IS. 1992. RNA sequence variants in live poliovirus vaccine and their relation to neurovirulence. 7. Virol. 66:966-70

29. Cochi SL, Sutter RW, Kew OM, Pallansch MA, Dowdle WR. 1997. A decision tree for stopping polio immunization. Techn. Consult. Global Erad. Poliomyelitis. Rep. EPI/POLIO/TECH.97/WP18. Geneva: WHO

30. Cooper PD. 1968. A genetic map of poliovirus temperature-sensitive mutants. Virology $35: 584-96$ 
31. Crainic R, Couillin P, Blondel B, Cabau N, Boue A, Horodniceanu F. 1983. Natural variation of poliovirus neutralization epitopes. Infect. Immun. 41:1217-25

32. Cruz RR. 1984. Cuba: mass polio vaccination program, 1962-1982. Rev. Infect. Dis. 6(Suppl. 2):S408-12

33. Cuervo NS, Guillot S, Romanenkova N, Combiescu M, Aubert-Combiescu A, et al. 2001. Genomic features of intertypic recombinant Sabin poliovirus strains excreted by primary vaccinees. 7. Virol. 75:5740-51

34. da Silva EE, da Costa EV, Kew OM. 2000. OPV-derived polioviruses isolated from recent cases of acute flaccid paralysis in Brazil are closely related to the prototype Sabin strains. Presented at Progr. Polio Eradication: Vaccine Strategies End Game, Paris, 28-30 June

35. De L, Nottay BK, Yang C-F, Holloway BP, Pallansch MA, Kew O. 1995. Identification of vaccine-related polioviruses by hybridization with specific RNA probes. F. Clin. Microbiol. 33:562-71

36. De L, Yang C-F, da Silva E, Boshell J, Cáceres P, et al. 1997. Genotype-specific RNA probes for the direct identification of wild polioviruses by blot hybridization. F. Clin. Microbiol. 35:2834-40

37. Delbrück M. 1958. Bacteriophage genetics. In Poliomyelitis: Papers and Discussion Presented at the Fourth International Poliomyelitis Conference, ed. IP Conference, pp. 342-53. Philadelphia: Lippincott

38. de Quadros CA, Andrus JK, Olivé JM, Guerra de Macedo C, Henderson DA. 1992. Polio eradication from the Western Hemisphere. Annu. Rev. Public Health 13:23952

39. Deshpande JM, Shetty SJ, Siddiqui ZA. 2003. Environmental surveillance system to track wild poliovirus transmission. Appl. Environ. Microbiol. 69:2919-27

40. Doi Y, Abe S, Yamamoto H, Horie H, Ohyama H, et al. 2001. Progress with inactivated poliovirus vaccines derived from the Sabin strains. Dev. Biol. 105:163-69

41. Domingo E, Holland JJ. 1997. RNA virus mutations and fitness for survival. Annu. Rev. Microbiol. 51:151-78

42. Dowdle WR, Birmingham ME. 1997. The biologic principles of poliovirus eradication. 7. Infect. Dis. 175(Suppl. 1):S286-92

43. Dowdle WR, de Gourville E, Kew OM, Pallansch MA, Wood DJ. 2003. Polio eradication: the OPV paradox. Rev. Med. Virol. 13:277-91

44. Dowdle WR, Wolff C, Sanders R, Lambert S, Best M. 2004. Will containment of wild poliovirus in laboratories and inactivated poliovirus vaccine production sites be effective for global certification? Bull. WHO 82:59-62

45. Drake JW, Holland JJ. 1999. Mutation rates among RNA viruses. Proc. Natl. Acad. Sci. USA 96:13910-13

46. Duintjer Tebbens RJ, Pallansch MA, Kew OM, Cáceres VM, Jafari H, et al. 2005. Risks of paralytic disease due to wild or vaccine-derived poliovirus after eradication. Risk Assess. In press

47. Ehrenfeld E, Teterina NL. 2002. Initiation of translation of picornavirus RNAs: structure and function of the internal ribosome entry site. See Ref. 175a, pp. 15969

48. El Bassioni L, Barakat I, Nasr E, de Gourville EM, Hovi T, et al. 2003. Prolonged detection of indigenous wild polioviruses in sewage from communities in Egypt. Am. 7 . Epidemiol. 158:807-15

49. Enders J, Weller T, Robbins F. 1949. Cultivation of the Lansing strain of poliomyelitis virus in cultures of various human embryonic tissues. Science 109:85-87 
50. Enders JF, Weller TH, Robbins FC. 1952. Alterations in pathogenicity for monkeys of Brunhilde strain of poliomyelitis virus following cultivation in human tissues. Fed. Proc. 11:467

51. Esteves K. 1988. Safety of oral poliomyelitis vaccine: results of a WHO enquiry. Bull. WHO 66:739-46

52. Fenner F, Henderson DA, Arita I, Ježek Z, Ladnyi ID. 1988. Smallpox and Its Eradication. Geneva: WHO

53. Ferguson M, Wood DJ, Minor PD. 1993. Antigenic structure of poliovirus in inactivated vaccines. 7. Gen. Virol. 74:685-90

54. Filman DJ, Syed R, Chow M, Macadam AJ, Minor PD, Hogle JM. 1989. Structural factors that control conformational transitions and serotype specificity in type 3 poliovirus. EMBO 7. 8:1567-79

55. Fine PEM, Carneiro IAM. 1999. Transmissibility and persistence of oral polio vaccine viruses: implications for the global poliomyelitis eradication initiative. Am. F. Epidemiol. 150:1001-21

56. Fine PEM, Oblapenko G, Sutter RW. 2004. Polio control after certification: major issues outstanding. Bull. WHO 82:47-52

57. FDA. 1991. Additional standards for viral vaccines; poliovirus vaccine live oral; final rule (21 CFR Part 630). Fed. Regist. 56:21418-38

58. Fricks CE, Icenogle JP, Hogle JM. 1985. Trypsin sensitivity of the Sabin strain type 1 poliovirus: cleavage sites in virions and related particles. F. Virol. 54:856-59

59. Furione M, Guillot S, Otelea D, Balanant J, Candrea A, Crainic R. 1993. Polioviruses with natural recombinant genomes isolated from vaccine-associated poliomyelitis. $\mathrm{Vi}$ rology 196:199-208

60. Gavrilin GV, Cherkasova EA, Lipskaya GY, Kew OM, Agol VI. 2000. Evolution of circulating wild poliovirus and of vaccine-derived poliovirus in an immunodeficient patient: a unifying model. F. Virol. 74:7381-90

61. Georgescu MM, Balanant J, Macadam A, Otelea D, Combiescu M, et al. 1997. Evolution of the Sabin type 1 poliovirus in humans: characterization of strains isolated from patients with vaccine-associated paralytic poliomyelitis. F. Virol. 71:7758-68

62. Gromeier M, Alexander L, Wimmer E. 1996. Internal ribosomal entry site substitution eliminates neurovirulence in intergeneric poliovirus recombinants. Proc. Natl. Acad. Sci. USA 93:2370-75

63. Gromeier M, Bossert B, Arita M, Nomoto A, Wimmer E. 1999. Dual stem loops within the poliovirus internal ribosome entry site controls neurovirulence. F. Virol. 73:95864

64. Gromeier M, Nomoto A. 2002. Determinants of poliovirus pathogenesis. See Ref. 175a, pp. 367-79

65. Guest S, Pilipenko E, Sharma K, Chumakov K, Roos RP. 2004. Molecular mechanisms of attenuation of the Sabin strain of poliovirus type 3. F. Virol. 78:11097-107

66. Guillot S, Caro V, Cuervo N, Korotkova E, Combiescu M, et al. 2000. Natural genetic exchanges between vaccine and wild poliovirus strains in humans. F. Virol. 74:843443

67. Hadler SC, Cochi SL, Bilous J, Cutts FT. 2004. Vaccination programs in developing countries. See Ref. 151a, pp. 1407-41

68. Haller AA, Stewart SR, Semler BL. 1996. Attenuation stem-loop lesions in the $5^{\prime}$ noncoding region of poliovirus RNA: neuronal cell-specific translation defects. 7. Virol. 70:1467-74 
69. Halsey NA, Pinto J, Espinosa-Rosales F, Faure-Fontenia MA, da Silva E, et al. 2004. Search for poliovirus carriers in persons with primary immune deficiency diseases in the United States, Mexico, Brazil, and the United Kingdom. Bull. WHO 82:38

70. Hammon WM, Coriell LL, Wehrle PF, Stokes J Jr. 1953. Evaluation of Red Cross gamma globulin as a prophylactic agent for poliomyelitis. IV. Final report of results based on clinical diagnoses. $7 A M A$ 151:1272-85

71. Hara M, Saito Y, Komatsu T, Kodama H, Abo W, et al. 1981. Antigenic analysis of polioviruses isolated from a child with agammaglobulinemia and paralytic poliomyelitis after Sabin vaccine administration. Microbiol. Immunol. 25:905-13

72. He Y-N, Bowman VD, Mueller S, Bator CM, Bella J, et al. 2000. Interaction of the poliovirus receptor with poliovirus. Proc. Natl. Acad. Sci. USA 97:79-84

73. Henderson DA, Borio LL, Lane JM. 2004. Smallpox and vaccinia. See Ref. 151a, pp. $123-53$

74. Hennessey KA, Lago H, Diomande F, Akoua-Koffi C, Cáceres VM, et al. 2005. Poliovirus vaccine shedding among persons with HIV, Côte d'Ivorie. F. Infect. Dis. In press

75. Hidalgo S, Garcia Erro M, Cisterna D, Freire MC. 2003. Paralytic poliomyelitis caused by a vaccine-derived polio virus in an antibody-deficient Argentinean child. Pediatr. Infect. Dis. 7. 22:570-72

76. Hogle JM. 2002. Poliovirus cell entry: common structural themes in viral cell entry pathways. Annu. Rev. Microbiol. 56:677-702

77. Hogle JM, Chow M, Filman DJ. 1985. The three-dimensional structure of poliovirus at 2.9 A resolution. Science 229:1358-65

78. Horstmann DM, McCollum RW, Mascola AD. 1954. Viremia in human poliomyelitis. 7. Exp. Med. 99:355-69

79. Hovi T, Stenvik M. 1994. Selective isolation of poliovirus in recombinant murine cell line expressing the human poliovirus receptor gene. 7. Clin. Microbiol. 32:136668

80. Hull HF, Ward NA, Milstien JB, de Quadros C. 1994. Paralytic poliomyelitis: seasoned strategies, disappearing disease. Lancet 343:1331-37

81. Iizuka N, Kohara M, Hagino-Yamagishi K, Abe S, Komatsu T, et al. 1989. Construction of less neurovirulent polioviruses by introducing deletions into the $5^{\prime}$ noncoding region of the genome. F. Virol. 63:5354-63

82. Joce R, Wood D, Brown D, Begg N. 1992. Paralytic poliomyelitis in England and Wales, 1985-91. Br. Med. 7. 305:79-82

83. Kawamura N, Kohara M, Abe S, Komatsu T, Tago K, et al. 1989. Determinants in the 5' noncoding region of poliovirus Sabin 1 RNA that influence the attenuation phenotype. 7. Virol. 63:1302-9

84. Kew OM, Morris-Glasgow V, Landaverde M, Burns C, Shaw J, et al. 2002. Outbreak of poliomyelitis in Hispaniola associated with circulating type 1 vaccine-derived poliovirus. Science 296:356-59

85. Kew OM, Mulders MN, Lipskaya GY, da Silva EE, Pallansch MA. 1995. Molecular epidemiology of polioviruses. Semin. Virol. 6:401-14

86. Kew OM, Nottay BK. 1984. Molecular epidemiology of polioviruses. Rev. Infect. Dis. 6(Suppl. 2):S499-504

87. Kew OM, Pallansch MA. 2002. The mechanism of polio eradication. See Ref. 175a, pp. 481-91 
88. Kew OM, Sutter RW, Nottay B, McDonough M, Prevots DR, et al. 1998. Prolonged replication of a type 1 vaccine-derived poliovirus in an immunodeficient patient. 7 . Clin. Microbiol. 36:2893-99

89. Kew OM, Wright PF, Agol VI, Delpeyroux F, Shimizu H, et al. 2004. Circulating vaccine-derived polioviruses: current state of knowledge. Bull. WHO 82:16-23

90. Khetsuriani N, Prevots DR, Quick L, Elder ME, Pallansch M, et al. 2003. Persistence of vaccine-derived polioviruses among immunodeficient persons with vaccine-associated paralytic poliomyelitis. F. Infect. Dis. 188:1845-52

91. Kilpatrick DR, Ching K, Iber J, Campagnoli R, Freeman CJ, et al. 2004. Multiplex PCR method for identifying recombinant vaccine-related polioviruses. F. Clin. Microbiol. 42:4313-15

92. Kilpatrick DR, Nottay B, Yang C-F, Yang S-J, da Silva E, et al. 1998. Serotypespecific identification of polioviruses by PCR using primers containing mixed-base or deoxyinosine residues at positions of codon degeneracy. 7. Clin. Microbiol. 36:35257

93. Kilpatrick DR, Nottay B, Yang C-F, Yang S-J, Mulders MN, et al. 1996. Group-specific identification of polioviruses by PCR using primers containing mixed-base or deoxyinosine residues at positions of codon degeneracy. F. Clin. Microbiol. 34:2990-96

94. Kim-Farley R, Bart K, Schonberger L, Orenstein W, Hinman A, et al. 1984. The epidemiology of poliomyelitis in the United States: virtual elimination of disease caused by wild virus. Lancet $2: 315-17$

95. Kinnunen L, Huovilainen A, Pöyry T, Hovi T. 1990. Rapid molecular evolution of wild type 3 poliovirus during infection in individual hosts. 7. Gen. Virol. 71:317-24

96. Kitamura N, Semler BL, Rothberg PG, Larsen GR, Adler CJ, et al. 1981. Primary structure, gene organization and polypeptide expression of poliovirus RNA. Nature 291:54753

96a. Knipe DM, Howley PM, Griffin DE, Lamb RA, Martin MA, et al., eds. 2001. Field's Virology. Philadelphia: Lippincott Williams \& Wilkins

97. Kohara M, Abe S, Komatsu T, Tago K, Arita M, Nomoto A. 1988. A recombinant virus between the Sabin 1 and Sabin 3 vaccine strains of poliovirus as a possible candidate for a new type 3 poliovirus live vaccine strain. F. Virol. 62:2828-35

98. Kohler KA, Banerjee K, Hlady GW, Andrus JK, Sutter RW. 2002. Vaccine-associated paralytic poliomyelitis in India during 1999: decreased risk despite massive use of oral polio vaccine. Bull. WHO 80:210-16

99. Koprowski H, Jervis GA, Norton TW. 1952. Immune responses in human volunteers upon oral administration of a rodent-adapted strain of poliomyelitis virus. Am. F. Hyg. 55:108-26

100. Korotkova EA, Park R, Cherkasova EA, Lipskaya GY, Chumakov KM, et al. 2003. Retrospective analysis of a local cessation of vaccination against poliomyelitis: a possible scenario for the future. 7. Virol. 77:12460-65

101. Kostrewski J, Kulesza A, Abgarowicz A. 1970. The epidemic of type 3 poliomyelitis in Poland in 1968. Epidemiol. Rev. 24:89-103

102. Kuechler E, Seipelt J, Liebig H-D, Sommergruber W. 2002. Picornavirus proteinasemediated shutoff of host cell translation: direct cleavage of a cellular initiation factor. See Ref. 175a, pp. 301-11

103. Lentz KN, Smith AD, Geisler SC, Cox S, Buontempo P, et al. 1997. Structure of poliovirus type 2 Lansing complexed with antiviral agent SCH48973: comparison of the structural and biological properties of three poliovirus serotypes. Structure 5:961-78 
104. Li CP, Schaeffer M. 1954. Isolation of a non-neurotropic variant of type 1 poliomyelitis virus. Proc. Soc. Exp. Biol. Med. 87:148-53

105. Lipskaya GY, Chervonskaya EA, Belova GI, Maslova SV, Kutateladze TN, et al. 1995. Geographic genotypes (geotypes) of poliovirus case isolates from the former Soviet Union: relatedness to other known poliovirus genotypes. 7. Gen. Virol. 76:168799

106. Lipskaya GY, Muzychenko AR, Kutitova OK, Maslova SV, Equestre M, et al. 1991. Frequent isolation of intertypic poliovirus recombinants with serotype 2 specificity from vaccine-associated polio cases. 7. Med. Virol. 35:290-96

107. Liu H-M, Zheng D-P, Zhang L-B, Oberste MS, Kew OM, Pallansch MA. 2003. Serial recombination during circulation of type 1 wild-vaccine recombinant polioviruses in China. F. Virol. 77:10994-1005

108. Liu H-M, Zheng D-P, Zhang L-B, Oberste MS, Pallansch MA, Kew OM. 2000. Molecular evolution of a type 1 wild-vaccine poliovirus recombinant during widespread circulation in China. 7. Virol. 74:11153-61

109. Macadam AJ, Arnold C, Howlett J, John A, Marsden S, et al. 1989. Reversion of the attenuated and temperature-sensitive phenotypes of the Sabin type 3 strain of poliovirus in vaccinees. Virology 172:408-14

110. Macadam AJ, Ferguson G, Stone DM, Meredith J, Almond JW, Minor PD. 2001. Liveattenuated strains of improved genetic stability. Dev. Biol. 105:179-87

111. Macadam AJ, Pollard SR, Ferguson G, Skuce R, Wood D, et al. 1993. Genetic basis of attenuation of the Sabin type 2 vaccine strain of poliovirus in primates. Virology 192:1826

112. MacCallum FO. 1971. Hypogammaglobulinaemia in the United Kingdom. VII. The role of humoral antibodies in the protection against and recovery from bacterial and virus infections in hypogammaglobulinaemia. Spec. Rep. Ser. Med. Res. Counc. 310:7285

113. MacLennan C, Dunn G, Huissoon AP, Kumararatne DS, Martín J, et al. 2004. Failure to clear persistent vaccine-derived neurovirulent poliovirus infection in an immunodeficient man. Lancet 363:1509-13

114. Majiyagbe J. 2004. The volunteers' contribution to polio eradication. Bull. WHO 82:2

115. Manor Y, Handsher R, Halmut T, Neuman M, Abramovitz B, et al. 1999. A doubleselective tissue culture system for isolation of wild-type poliovirus from sewage applied in a long-term environmental surveillance. Appl. Environ. Microbiol. 65:94-97

116. Manor Y, Handsher R, Halmut T, Neuman M, Bobrov A, et al. 1999. Detection of poliovirus circulation by environmental surveillance in the absence of clinical cases in Israel and the Palestinian Authority. F. Clin. Microbiol. 37:1670-75

117. Martín J, Dunn G, Hull R, Patel V, Minor PD. 2000. Evolution of the Sabin strain of type 3 poliovirus in an immunodeficient patient during the entire 637-day period of virus excretion. F. Virol. 74:3001-10

118. Martín J, Ferguson GL, Wood DJ, Minor PD. 2000. The vaccine origin of the 1968 epidemic of type 3 poliomyelitis in Poland. Virology 278:42-49

119. Martín J, Odoom K, Tuite G, Dunn G, Hopewell N, et al. 2004. Long-term excretion of vaccine-derived poliovirus by a healthy child. F. Virol. 78:13839-47

120. Más Lago P. 1999. Eradication of poliomyelitis in Cuba: a historical perspective. Bull. WHO 77:681-87

121. Melnick JL. 1978. Advantages and disadvantages of the killed and live poliomyelitis vaccines. Bull. WHO 56:21-38 
122. Mendelsohn CL, Wimmer E, Racaniello VR. 1989. Cellular receptor for poliovirus: molecular cloning, nucleotide sequence, and expression of a new member of the immunoglobulin superfamily. Cell 56:855-65

123. Minor P. 2001. Characteristics of poliovirus strains from long-term excretors with primary immunodeficiencies. Dev. Biol. 105:75-80

124. Minor PD. 1990. Antigenic structure of picornaviruses. Curr: Top. Microbiol. Immunol. $161: 121-54$

125. Minor PD. 2004. Polio eradication, cessation of vaccination and re-emergence of disease. Nat. Rev. Microbiol. 2:473-82

126. Minor PD, Almond JW. 2002. Poliovirus vaccines: molecular biology and immune response. See Ref. 175 a, pp. 381-90

127. Minor PD, Dunn G. 1988. The effect of sequences in the $5^{\prime}$ non-coding region on the replication of polioviruses in the human gut. F. Gen. Virol. 69:1091-96

128. Minor PD, Dunn G, Evans DMA, Magrath DI, John A, et al. 1989. The temperature sensitivity of the Sabin type 3 vaccine strain of poliovirus: molecular and structural effects of a mutation in the capsid protein VP3. 7. Gen. Virol. 70:1117-23

129. Minor PD, John A, Ferguson M, Icenogle JP. 1986. Antigenic and molecular evolution of the vaccine strain of type 3 poliovirus during the period of excretion by a primary vaccinee. 7. Gen. Virol. 67:693-706

130. Misbah SA, Lawrence PA, Kurtz JB, Chapel HM. 1991. Prolonged faecal excretion of poliovirus in a nurse with common variable hypogammaglobulinaemia. Postgrad. Med. 7. $67: 301-3$

130a. Mueller S, Wimmer E. 2003. Recruitment of nectin-3 to cell-cell junctions through trans-heterophilic interaction with CD155, a vitronectin and poliovirus receptor that localizes to alpha(v)beta3integrin-containing membrane microdomains. F. Biol. Chem. 278:31251-60

131. Mulders MN, van Loon AM, van der Avoort HGAM, Riemerink JHJ, Ras A, et al. 1995. Molecular characterization of the wild poliovirus type 3 epidemic in the Netherlands (1992-93). 7. Clin. Microbiol. 33:3252-56

132. Muzychenko AR, Lipskaya GY, Maslova SV, Svitkin YV, Pilipenko EV, et al. 1991. Coupled mutations in the $5^{\prime}$-untranslated region of the Sabin poliovirus strains during in vivo passage: structural and functional implications. Virus Res. 21:111-22

133. Nakano JH, Hatch MH, Thieme ML, Nottay B. 1978. Parameters for differentiating vaccine-derived and wild poliovirus strains. Prog. Med. Virol. 24:78-206

134. Nathanson N, Langmuir AD. 1963. The Cutter Incident. Poliomyelitis following formaldehyde-inactivated poliovirus vaccination in the United States in the spring of 1955. I. Background. Am. 7. Hyg. 78:16-28

135. Nathanson N, Martin JR. 1979. The epidemiology of poliomyelitis: enigmas surrounding its appearance, epidemicity, and disappearance. Am. F. Epidemiol. 110:67292

136. Nomoto A, Arita I. 2002. Eradication of poliomyelitis. Nat. Immunol. 3:205-8

137. Nomoto A, Iizuka N, Kohara M, Arita M. 1988. Strategy for construction of live picornavirus vaccines. Vaccine 6:134-37

138. Nomoto A, Omata T, Toyoda H, Kuge S, Horie H, et al. 1982. Complete nucleotide sequence of the attenuated poliovirus Sabin 1 strain genome. Proc. Natl. Acad. Sci. USA 79:5793-97

139. Oker-Blom N, Penttinen K, Weckström P. 1984. Inactivated poliovirus vaccine in Finland. Rev. Infect. Dis. 6(Suppl. 2):S461-62 
140. Omata T, Kohara M, Kuge S, Komatsu T, Abe S, et al. 1986. Genetic analysis of the attenuation phenotype of poliovirus type 1.7. Virol. 58:348-58

141. Page GS, Mosser AG, Hogle JM, Filman DJ, Rueckert RR, Chow M. 1988. Threedimensional structure of poliovirus serotype 1 neutralizing determinants. F. Virol. 62:1781-94

142. Pallansch MA, Roos RP. 2001. Enteroviruses: polioviruses, coxsackieviruses, echoviruses, and newer enteroviruses. See Ref. 96a, pp. 723-75

143. Pan Am. Health Organ. 1959. Proc. 1st Int. Conf. Live Poliovirus Vaccines. Washington, DC: Pan Am. Health Organ.

144. Pan Am. Health Organ. 1960. Proc. 2nd Int. Conf. Live Poliovirus Vaccines. Washington, DC: Pan Am. Health Organ.

145. Pan Am. Health Organ. 1985. Director announces campaign to eradicate poliomyelitis from the Americas by 1990. Bull. Pan Am. Health Organ. 19:21-35

146. Park RE, Yang C-F, Yang S-J, Asghar H, Nategh R, Kew OM. 2002. Molecular characterization of divergent Sabin 2-derived isolates from patients with paralysis. Rep. P13-3, Am. Soc. Virol. 21st Annu. Meet., Lexington, Kentucky

147. Patriarca PA, Sutter RW, Oostvogel PM. 1997. Outbreaks of paralytic poliomyelitis, 1976-1995.7. Infect. Dis. 175(Suppl. 1):S165-72

148. Paul AV, Mugavero J, Yin J, Hobson S, Schultz S, et al. 2000. Studies on the attenuation phenotype of polio vaccines: poliovirus RNA polymerase derived from Sabin type 1 sequence is temperature sensitive in the uridylylation of VPg. Virology 272:7284

149. Paul JR. 1971. A History of Poliomyelitis. New Haven, CT: Yale Univ. Press

150. Payne AMM. 1961. Field safety and efficacy of live attenuated poliovirus vaccines. In $P_{0-}$ liomyelitis: Papers and Discussion Presented at the Fifth International Poliomyelitis Conference. Philadelphia: Lippincott

151. Pipkin PA, Wood DJ, Racaniello VR, Minor PD. 1993. Characterisation of L cells expressing the human poliovirus receptor for the specific detection of polioviruses in vitro. 7. Virol. Methods 41:333-40

151a. Plotkin SA, Orenstein WA, eds. 2004. Vaccines. Philadelphia: Saunders

152. Plotkin SA, Vidor E. 2004. Poliovirus vaccine: inactivated. See Ref. 151a, pp. 62549

153. Plotkin SL, Plotkin SA. 2004. A short history of vaccination. See Ref. 151a, pp. 1-15

154. Pollard SR, Dunn G, Cammack N, Minor PD, Almond JW. 1989. Nucleotide sequence of a neurovirulent variant of the type 2 oral poliovirus vaccine. F. Virol. 63:494951

155. Pöyry T, Stenvik M, Hovi T. 1988. Viruses in sewage waters during and after a poliomyelitis outbreak and subsequent nationwide oral poliovirus vaccination campaign in Finland. Appl. Environ. Microbiol. 54:371-74

156. Racaniello VR. 2001. Picornaviridae: the viruses and their replication. See Ref. 96a, pp. $685-722$

157. Racaniello VR, Baltimore D. 1981. Cloned poliovirus complementary DNA is infectious in mammalian cells. Science 214:916-19

158. Racaniello VR, Baltimore D. 1981. Molecular cloning of poliovirus cDNA and determination of the complete nucleotide sequence of the viral genome. Proc. Natl. Acad. Sci. USA 78:4887-91

159. Racaniello VR, Ren R. 1996. Poliovirus biology and pathogenesis. Curr. Top. Microbiol. Immunol. 206:305-25 
160. Ren R, Moss EG, Racaniello VR. 1991. Identification of two determinants that attenuate vaccine-related type 2 poliovirus. F. Virol. 65:1377-82

161. Rezapkin GV, Chumakov KM, Lu Z, Ran Y, Dragunsky EM, Levenbook IS. 1994. Microevolution of Sabin 1 strain in vitro and genetic stability of oral poliovirus vaccine. Virology 202:370-78

162. Rezapkin GV, Douthitt M, Dragunsky E, Chumakov KM. 1999. Reevaluation of nucleotide sequences of wild-type and attenuated polioviruses of type 3. Virus Res. 65:11119

163. Rezapkin GV, Fan L, Asher DM, Fibi MR, Dragunsky EM, Chumakov KM. 1999. Mutations in Sabin 2 strain of poliovirus and stability of attenuated phenotype. Virology 258:152-60

164. Rico-Hesse R, Pallansch MA, Nottay BK, Kew OM. 1987. Geographic distribution of wild poliovirus type 1 genotypes. Virology 160:311-22

165. Risi JB. 1984. The control of poliomyelitis in Brazil. Rev. Infect. Dis. 6(Suppl. 2):S4003

166. Robbins FC. 2004. The history of polio vaccine development. See Ref. 151a, pp. 1730

167. Roberts L. 2005. Infectious disease. Polio eradication effort adds new weapon to its armory. Science 307:190

168. Rosen FS, Cooper MD, Wedgwood RJP. 1995. The primary immunodeficiencies. $N$. Engl. 7. Med. 333:431-40

169. Rousset D, Rakoto-Andrianarivelo M, Razafindratsimandresy R, Randriamanalina B, Guillot S, et al. 2003. Recombinant vaccine-derived poliovirus in Madagascar. Emerg. Infect. Dis. 9:885-87

170. Sabin AB. 1985. Oral poliovirus vaccine: history of its development and use, and current strategies to eliminate poliomyelitis from the world. F. Infect. Dis. 151:420-36

171. Sabin AB, Boulger LR. 1973. History of Sabin attenuated poliovirus oral live vaccine strains. 7. Biol. Stand. 1:115-18

172. Sabin AB, Ramos-Alvarez M, Alvarez-Amezquita J, Pelon W, Michaels RH, et al. 1960. Live, orally given poliovirus vaccine. Effects of rapid mass immunization on population under conditions of massive enteric infection with other viruses. FAMA 173:1521-26

173. SalkJE, Krech U, Youngner JS, Bennett BL, Lewis LJ, Bazeley PL. 1954. Formaldehyde treatment and safety testing of experimental poliomyelitis vaccines. Am. F. Public Health 44:563-70

174. Savilahti E, Klemola T, Carlsson B, Mellander L, Stenvik M, Hovi T. 1988. Inadequacy of mucosal IgM antibodies in selective IgA deficiency: excretion of attenuated polio viruses is prolonged. F. Clin. Immunol. 8:89-94

175. Schonberger LB, Sullivan-Bolyai JZ, Bryan JA. 1978. Poliomyelitis in the United States. Adv. Neurol. 19:217-27

175a. Semler BL, Wimmer E, eds. 2002. Molecular Biology of Picornaviruses. Washington, DC: ASM Press

176. Sever J. 2001. Rotary International: a partner in polio eradication. Dev. Biol. 105:105-8

177. Shimizu H, Thorley B, Paladin FJ, Brussen KA, Stambos V, et al. 2004. Circulation of type 1 vaccine-derived poliovirus in the Philippines in 2001. 7. Virol. 78:13512-21

178. Shimojo H. 1984. Poliomyelitis control in Japan. Rev. Infect. Dis. 6(Suppl. 2):S427-S30

179. Shulman LM, Handsher R, Yang S-J, Yang C-F, Manor J, et al. 2000. Resolution of the pathways of poliovirus type 1 transmission during an outbreak. 7 . Clin. Microbiol. 38:945-52 
180. Shulman LM, Manor J, Handsher R, Delpeyroux F, McDonough MJ, et al. 2000. Molecular and antigenic characterization of a highly evolved derivative of the type 2 oral poliovaccine strain isolated from sewage in Israel. 7. Clin. Microbiol. 38:372934

181. Smith J, Leke R, Adams A, Tangermann RH. 2004. Certification of polio eradication: process and lessons learned. Bull. WHO 82:24-30

182. Stanway G, Hughes PJ, Mountford RC, Reeve P, Minor PD, et al. 1984. Comparison of the complete nucleotide sequences of the genomes of the neurovirulent poliovirus P3/Leon/37 and its attenuated Sabin vaccine derivative P3/Leon 12a1b. Proc. Natl. Acad. Sci. USA 81:1539-43

183. Strebel PM, Sutter RW, Cochi SL, Biellik RJ, Brink EW, et al. 1992. Epidemiology of poliomyelitis in the United States one decade after the last reported case of indigenous wild virus-associated disease. Clin. Infect. Dis. 14:568-79

184. Sutter RW, Cáceres VM, Más Lago P. 2004. The role of routine polio immunization in the post-certification era. Bull. WHO 82:31-39

185. Sutter RW, Kew OM, Cochi SL. 2004. Poliovirus vaccine: live. See Ref. 151a, pp. 651705

186. Sutter RW, Prevots R. 1994. Vaccine-associated paralytic poliomyelitis among immunodeficient persons. Infect. Med. 11:426-38

187. Sutter RW, Suleiman AJM, Malankar P, Al-Khusaiby S, Mehta F, et al. 2000. Trial of a supplemental dose of four poliovirus vaccines. N. Engl. F. Med. 343:767-73

188. Svitkin Y, Cammack N, Minor PD, Almond JW. 1990. Translation deficiency of the Sabin type 3 poliovirus genome: association with an attenuating mutation C472-U. Virology 175:103-9

189. Taffs RE, Chumakov KM, Rezapkin GV, Lu Z, Douthitt M, et al. 1995. Genetic stability and mutant selection in Sabin 2 strain of oral poliovirus vaccine grown under different cell culture conditions. Virology 209:366-73

190. Tambini G, Andrus JK, Marques E, Boshell J, Pallansch M, et al. 1993. Direct detection of wild poliovirus transmission by stool surveys of healthy children and analysis of community wastewater. 7 . Infect. Dis. 168:1510-14

191. Tardy-Panit M, Blondel B, Martin A, Tekaia F, Horaud F, Delpeyroux F. 1993. A mutation in the RNA polymerase of poliovirus type 1 contributes to attenuation in mice. 7. Virol. 67:4630-38

192. Tech. Consult. Group WHO Glob. Erad. Poliomyelitis. 2002. "Endgame" issues for the Global Polio Eradication Initiative. Clin. Infect. Dis. 34:72-77

193. Terry L. 1962. The association of cases of poliomyelitis with the use of type 3 oral poliomyelitis vaccines. U.S. Dep. Health, Educ. Welf., Washington, DC

194. Theiler M. 1946. A mutant strain of the Lansing virus. Presented at Mech. Immun. Poliomyelitis, Baltimore, $\mathrm{MD}$

195. Theiler M, Smith HH. 1937. The effect of prolonged cultivation in vitro upon the pathogenicity of yellow fever virus. 7. Exp. Med. 65:767-86

196. Toyoda H, Kohara M, Kataoka Y, Suganuma T, Omata T, et al. 1984. Complete nucleotide sequences of all three poliovirus serotype genomes: implication for genetic relationship, gene function and antigenic determinants. F. Mol. Biol. 174:56185

197. van der Avoort HG, ReimerinkJH, Ras A, Mulders MN, van Loon AM. 1995. Isolation of epidemic poliovirus from sewage during the 1992-3 type 3 outbreak in The Netherlands. Epidemiol. Infect. 114:481-91 
198. van der Avoort HGAM, Hull BP, Hovi T, Pallansch MA, Kew OM, et al. 1995. A comparative study of five methods of intratypic differentiation of polioviruses. 7 . Clin. Microbiol. 33:2562-66

199. van Wezel AL, Hazendonk AG. 1979. Intratypic serodifferentiation of poliomyelitis virus by strain-specific antisera. Intervirology 11:2-8

200. van Wezel AL, van Steenis G, van der Marel P, Osterhaus AD. 1984. Inactivated poliovirus vaccine: current production methods and new developments. Rev. Infect. Dis. 6(Suppl. 2):S335-40

201. Varughese PV, Carter AO, Acres SE, Furesz J. 1989. Eradication of indigenous poliomyelitis in Canada: impact of immunization strategies. Can. F. Public Health 80:36368

202. Vonka V, Janda Z, Simon J, Adam E, Starek M. 1967. A new type 3 attenuated poliovirus for possible use in oral poliovirus vaccine. Prog. Med. Virol. 9:204-55

203. Westrop GD, Wareham KA, Evans DM, Dunn G, Minor PD, et al. 1989. Genetic basis of attenuation of the Sabin type 3 oral poliovirus vaccine. 7. Virol. 63:133844

204. WHO Consult. Group. 1982. The relation between acute persisting spinal paralysis and poliomyelitis vaccine: results of a ten-year enquiry. Bull. WHO 60:231-42

205. Wimmer E, Hellen CU, Cao X. 1993. Genetics of poliovirus. Annu. Rev. Genet. 27:353436

206. Wood DJ, Sutter RW, Dowdle WR. 2000. Stopping poliovirus vaccination after eradication: issues and challenges. Bull. WHO 78:347-57

207. World Health Assembly. 1988. Polio eradication by the year 2000. Rep. Resolution 41.28. Geneva: WHO

208. WHO. 2001. Transmission of wild poliovirus type 2: apparent global interruption. Wkly. Epidemiol. Rec. 76:95-97

209. WHO. 2002. Expanding contributions of the Global Laboratory Network for Poliomyelitis Eradication. Wkly. Epidemiol. Rec. 77:133-37

210. WHO. 2002. Progress towards the global eradication of poliomyelitis, 2001. Wkly. Epidemiol. Rec. 77:98-107

211. WHO. 2003. Conclusions and recommendations: WHO informal consultation on identification and management of vaccine-derived polioviruses. Geneva: WHO

212. WHO. 2003. Global action plan for laboratory containment of wild polioviruses. Rep. WHO/V\&B/03.11. Geneva: WHO 2nd ed.

213. WHO. 2003. Global Polio Eradication Initiative, Strategic Plan 2004-2008. Geneva: WHO

214. WHO. 2003. Laboratory surveillance for wild and vaccine-derived polioviruses, January 2002-June 2003. Wkly. Epidemiol. Rec. 78:341-48

215. WHO. 2004. Global Polio Eradication Initiative, Strategic Plan 2004-2008. Wkly. Epidemiol. Rec. 79:55-57

216. WHO. 2004. Laboratory surveillance for wild and vaccine-derived polioviruses, January 2003-June 2004. Wkly. Epidemiol. Rec. 79:393-98

217. WHO. 2004. Polio laboratory manual, 4th edition. Geneva: WHO

218. WHO. 2004. Poliomyelitis: imported case in Sudan, situation in northern Nigeria. Wkly. Epidemiol. Rec. 79:245-46

219. WHO. 2004. Progress towards global eradication of poliomyelitis, 2003 and JanuaryApril 2004. Wkly. Epidemiol. Rec. 79:225-34

220. WHO. 2004. Progress towards global poliomyelitis eradication: preparation for the oral poliovirus vaccine cessation era. Wkly. Epidemiol. Rec. 79:349-56 
221. WHO. 2004. Special theme-polio eradication: end-stage challenges. Bull. WHO 82:170

222. WHO. 2005. Poliomyelitis outbreak escalates in the Sudan. Wkly. Epidemiol. Rec. 80:2-3

223. Yang C-F, Chen H-Y, Jorba J, Sun H-C, Yang S-J, et al. 2005. Recombination across multiple lineages of type 1 vaccine-derived poliovirus emerging during chronic infection of an immunodeficient patient. F. Virol. In press

224. Yang C-F, De L, Holloway BP, Pallansch MA, Kew OM. 1991. Detection and identification of vaccine-related polioviruses by the polymerase chain reaction. Virus Res. 20:159-79

225. Yang C-F, De L, Yang S-J, Gómez JR, Cruz JR, et al. 1992. Genotype-specific in vitro amplification of sequences of the wild type 3 polioviruses from Mexico and Guatemala. Virus Res. 24:277-96

226. Yang C-F, Naguib T, Yang S-J, Nasr E, Jorba J, et al. 2003. Circulation of endemic type 2 vaccine-derived poliovirus in Egypt, 1983 to 1993. 7. Virol. 77:8366-77

227. Yoneyama T, Hagiwara A, Hara M, Shimojo H. 1982. Alteration in oligonucleotide fingerprint patterns of the viral genome in poliovirus type 2 isolated from paralytic patients. Infect. Immun. 37:46-53

228. Yoshida H, Horie H, Matsuura K, Kitamura T, Hashizume S, Miyamura T. 2002. Prevalence of vaccine-derived polioviruses in the environment. F. Gen. Virol. 83:110711 
Annual Review of Microbiology

Volume 59, 2005

Frontispiece

Georges N. Cohen .......................................................... xiv

Looking Back

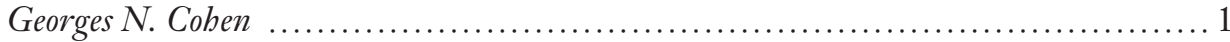

Signaling in the Arbuscular Mycorrhizal Symbiosis

Maria 7. Harrison

Interplay Between DNA Replication and Recombination in

Prokaryotes

Kenneth N. Kreuzer

Yersinia Outer Proteins: Role in Modulation of Host Cell Signaling

Responses and Pathogenesis

Gloria I. Viboud and James B. Bliska

Diversity and Evolution of Protein Translocation

Mechtbild Poblschröder, Enno Hartmann, Nicholas F. Hand, Kieran Dilks, and Alex Haddad

Alternative Candida albicans Lifestyles: Growth on Surfaces

Carol A. Kumamoto and Marcelo D. Vinces

Yeast Evolution and Comparative Genomics

Gianni Liti and Edward 7. Louis.

Biology of Bacteriocyte-Associated Endosymbionts of Plant

Sap-Sucking Insects

Paul Baumann

Genome Trees and the Nature of Genome Evolution

Berend Snel, Martijn A. Huynen, and Bas E. Dutilh

Cellular Functions, Mechanism of Action, and Regulation of FtsH

Protease

Koreaki Ito and Yoshinori Akiyama 
Mating in Candida albicans and the Search for a Sexual Cycle

R.F. Bennett and A.D. Fohnson

Applications of Autofluorescent Proteins for In Situ Studies in

Microbial Ecology

Estibaliz Larrainzar, Fergal O'Gara, and Fohn P. Morrissey

The Genetics of the Persistent Infection and Demyelinating Disease

Caused by Theiler's Virus

Michel Brahic, Jean-François Bureau, and Thomas Michiels

279

Intracellular Compartmentation in Planctomycetes

fohn A. Fuerst ....

Biogenesis of Inner Membrane Proteins in Escherichia coli

Foen Luirink, Gunnar von Heijne, Edith Houben, and Fan-Willem de Gier

Genome-Wide Responses to DNA-Damaging Agents

Rebecca C. Fry, Thomas 7. Begley, and Leona D. Samson

The Rcs Phosphorelay: A Complex Signal Transduction System

Nadim Majdalani and Susan Gottesman

Translational Regulation of GCN4 and the General Amino Acid

Control of Yeast

Alan G. Hinnebusch

Biogenesis, Architecture, and Function of Bacterial Type IV Secretion

Systems

Peter 7. Cbristie, Krishnamohan Atmakuri, Vidhya Krishnamoortby,

Simon Fakubowski, and Eric Cascales.

Regulation of Bacterial Gene Expression by Riboswitches

Wade C. Winkler and Ronald R. Breaker....

Opportunities for Genetic Investigation Afforded by Acinetobacter

baylyi, A Nutritionally Versatile Bacterial Species that is Highly

Competent for Natural Transformation

David M. Young, Donna Parke, and L. Nicholas Ornston

The Origins of New Pandemic Viruses: The Acquisition of New Host

Ranges by Canine Parvovirus and Influenza A Viruses

Colin R. Parrish and Yoshibiro Kawaoka

Vaccine-Derived Polioviruses and the Endgame Strategy for Global

Polio Eradication

Olen M. Kew, Roland W. Sutter, Esther M. de Gourville, Walter R. Dowdle, and Mark A. Pallansch 


\section{INDEXES}

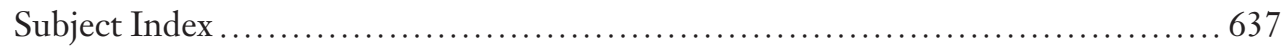

Cumulative Index of Contributing Authors, Volumes 55-59 ................... 675

Cumulative Index of Chapter Titles, Volumes 55-59 ............................ 678

\section{ERRATA}

An online log of corrections to Annual Review of Microbiology chapters

may be found at http://micro.annualreviews.org/ 